\title{
Clonal Evolution of TP53 c.375+1G>A Mutation in Pre- and Post- Neo-Adjuvant Chemotherapy (NACT) Tumor Samples in High-Grade Serous Ovarian Cancer (HGSOC)
}

\author{
Marica Garziera ${ }^{1, *(1)}$, Erika Cecchin ${ }^{1}\left(\mathbb{0}\right.$, Giorgio Giorda $^{2}$, Roberto Sorio ${ }^{3}$, Simona Scalone ${ }^{3}$, \\ Elena De Mattia ${ }^{1}{ }^{1}$, Rossana Roncato ${ }^{1}$, Sara Gagno ${ }^{1}$, Elena Poletto ${ }^{4}$, Loredana Romanato ${ }^{1}$, \\ Fabrizio Ecca ${ }^{1}$ D, Vincenzo Canzonieri ${ }^{5,6,+}$ (D) and Giuseppe Toffoli ${ }^{1,+}$ (D) \\ 1 Experimental and Clinical Pharmacology Unit, Centro di Riferimento Oncologico (CRO), IRCCS, \\ 33081 Aviano, Italy; ececchin@cro.it (E.C.); edemattia@cro.it (E.D.M.); rroncato@cro.it (R.R.); \\ sgagno@cro.it (S.G.); lromanato@cro.it (L.R.); fabrizio.ecca@cro.it (F.E.); gtoffoli@cro.it (G.T.) \\ Gynecological Oncology Unit, Centro di Riferimento Oncologico (CRO), IRCCS, 33081 Aviano, Italy; \\ ggiorda@cro.it \\ 3 Medical Oncology Unit C, Centro di Riferimento Oncologico (CRO), IRCCS, 33081 Aviano, Italy; \\ rsorio@cro.it (R.S.); sscalone@cro.it (S.S.) \\ 4 Medical Oncology, "Santa Maria della Misericordia” University Hospital, ASUIUD, 33100 Udine, Italy; \\ polettoelena@libero.it \\ 5 Pathology Unit, Centro di Riferimento Oncologico (CRO), IRCCS, 33081 Aviano, Italy; vcanzonieri@cro.it \\ 6 Department of Medical, Surgical and Health Sciences, University of Trieste, 34127 Trieste, Italy \\ * Correspondence: mgarziera@cro.it; Tel.: +39-0434-659-765 \\ + V. Canzonieri and G. Toffoli share last authorship.
}

Received: 20 August 2019; Accepted: 30 September 2019; Published: 1 October 2019

\begin{abstract}
Carboplatin/paclitaxel is the reference regimen in the treatment of advanced high-grade serous ovarian cancer (HGSOC) in neo-adjuvant chemotherapy (NACT) before interval debulking surgery (IDS). To identify new genetic markers of platinum-resistance, next-generation sequencing (NGS) analysis of 26 cancer-genes was performed on paired matched pre- and post-NACT tumor and blood samples in a patient with stage IV HGSOC treated with NACT-IDS, showing platinum-refractory/resistance and poor prognosis. Only the TP53 c.375+1G >A somatic mutation was identified in both tumor samples. This variant, associated with aberrant splicing, was in trans configuration with the 72Arg allele of the known germline polymorphism TP53 c.215C >G (p. Pro72Arg). In the post-NACT tumor sample we observed the complete expansion of the TP53 c. $375+1 \mathrm{G}>\mathrm{A}$ driver mutant clone with somatic loss of the treatment-sensitive 72Arg allele. NGS results were confirmed with Sanger method and immunostaining for p53, BRCA1, p16, WT1, and Ki-67 markers were evaluated. This study showed that (i) the splice mutation in TP53 was present as an early driver mutation at diagnosis; (ii) the mutational profile was shared in pre- and post-NACT tumor samples; (iii) the complete expansion of a single dominant mutant clone through loss of heterozygosity ( $\mathrm{LOH}$ ) had occurred, suggesting a possible mechanism of platinum-resistance in HGSOC under the pressure of NACT.
\end{abstract}

Keywords: HGSOC; NACT; NGS; TP53; chemoresistance

\section{Introduction}

Epithelial ovarian cancer is the most lethal malignancy of the female genital tract and the eighth leading cause of cancer-related death in 2018, among women worldwide [1]. High-grade serous 
ovarian cancer (HGSOC) accounts for up to $60-70 \%$ of ovarian carcinomas [2]. Most patients receive a diagnosis at an advanced stage (III-IV) of the disease because of a lack of specific symptoms, ending in a poor prognosis with a five-year survival rate of only $45 \%$ [3]. Despite an initial good response to platinum-based chemotherapy relapse seems unavoidable, with $~ 70 \%$ of patients experiencing disease recurrence in the first two years since diagnosis and requiring further treatments [4]. Primary debulking surgery (PDS), followed by platinum-based chemotherapy, is the standard treatment for advanced ovarian cancer, including HGSOC. Residual disease (RD) is a major prognostic factor for survival with no visible tumor residue $(\mathrm{RD}=0)$ as the goal of surgery [5]; nevertheless, a complete resection is very difficult to achieve in patients with massively disseminated tumors [6]. Neo-adjuvant chemotherapy (NACT) based on a platinum/taxane combination, prior to interval debulking surgery (IDS), is an alternative treatment option in patients with stage III-IV advanced ovarian cancer who are not candidates for PDS, due to unresectable disease and/or poor performance status $[7,8]$. The benefits from NACT-IDS, compared to PDS followed by standard chemotherapy, are still controversial. Although two randomized trials $[9,10]$ found an equivalent overall survival (OS) and reduced surgical morbidity and one study showed mortality reduction with NACT [11], in several other studies [8,12-14], less favorable outcomes, such as decreased OS, and an increased toxicity due to NACT, were reported when comparing the two treatment options. Furthermore, some studies have shown an increased risk of developing platinum resistance in stage III-IV ovarian cancer after NACT [6,8], and the in vitro induction of platinum and paclitaxel resistance after NACT and IDS, respectively $[15,16]$. The development of chemotherapy-refractory/resistant clones is a critical barrier to cure patients with advanced ovarian cancer, in particular HGSOC. However, relatively little is known of the genomic evolution of HGSOC under the selective pressure of chemotherapy. Although somatic mutations in TP53 are a frequent event in these patients $[17,18]$ and $\sim 20 \%$ are also mutated in the $B R C A 1 / 2$ susceptibility genes, due to a combination of germline and somatic mutations [19], extensive intratumoral heterogeneity in primary HGSOCs has been documented by the use of high-throughput sequencing techniques [20-23]. Next-generation sequencing (NGS) technology has expanded the knowledge of the complex genomic heterogeneity of ovarian cancer through the discovery of candidate targets for future therapeutic applications. In addition, the identification of germline and somatic mutations better define the subtype-specific molecular signatures, highlighting mechanisms of treatment resistance influencing ovarian and gynecologic malignancies [24]. This study, using targeted NGS, analyzed matched pre- and post-NACT ovarian tumor specimens and matched blood samples, from a patient with HGSOC characterized by a poor response to treatment and early death, within a 26 cancer-genes panel $[23,25]$. The aim was to compare tumor molecular profiles before and after NACT, based on the carboplatin/paclitaxel combination, in order to evaluate its influence in determining the platinum-refractory/resistance and outcomes in the HGSOC. A somatic mutation and a germline polymorphic variant coupled in trans configuration (i.e., located in different chromosomes) were identified in TP53 and also confirmed by standard Sanger sequencing. Through loss of heterozygosity ( $\mathrm{LOH}$, i.e., allelic imbalance or copy-neutral $\mathrm{LOH}$ ), we observed the expansion of the founder clone with the TP53 driver mutation which was coupled with the loss of the treatment-sensitive variant germline allele, suggesting a mechanism of chemoresistance in HGSOC. Moreover, to better characterize the HGSOC, immunostaining for $\mathrm{p} 53$ (in pre- and post-NACT tumor samples) and for BRCA1, p16, Wilms tumor 1 (WT1) and Ki-67 markers in chemo-naïve tumor, were also determined. Patients with trans configuration of the TP53 variants identified by NGS in the tumor are at high risk of mutation selection by NACT based on platinum/paclitaxel combination therapy. This study may have relevant impact in translational medicine and reveal a clinical applicability in the choice of treatment in HGSOC and advanced ovarian cancer. 


\section{Materials and Methods}

\subsection{Sample Collection and Human Ethics}

The patient included in this report was diagnosed and treated at CRO Institute between February 2005 and August 2007. Tumor and blood matched samples were collected at diagnostic laparoscopy (D-LPS) and IDS and retrospectively analyzed. Tumor staging and tumor grading were assessed according to Fédération Internationale de Ginécologie et d'Obstetrique (FIGO) and to WHO (World Health Organization) criteria respectively. Clinico-pathological characteristics, treatment, and complete follow-up information were collected from the medical record as current clinical surveillance procedures. OS was defined as the interval between diagnosis (at D-LPS) and the date of death. Time to recurrence (TTR) was defined as the interval between IDS and the date of the first recurrence/progression. Platinum-free interval (PFI) was defined as the interval between the end of the first-line platinum-based treatment after IDS and the date of first recurrence/progression. The patient was defined as "platinum-resistant" because relapse occurred within $<6$ months from the end of platinum treatment [23]. Clinical genetic testing of germline mutations in BRCA1/2 genes was not performed at the time of the enrollment because the patient had no family history of breast and/or ovarian cancer [23]. Written informed consent was obtained from the patient with histologically confirmed epithelial ovarian cancer for the use of peripheral blood, tissue samples, and clinical data for research purposes. The study was conducted in accordance with the Declaration of Helsinki and was approved by the Ethics Committee of the CRO Aviano National Cancer Institute, Italy (Institutional Review Board n. CRO-2014-43).

\subsection{Next Generation Sequencing Analysis and Somatic LOH Analysis}

Single nucleotide variants (SNVs) in TP53 for this patient with HGSOC emerged during a genomics screen aimed to identify new genetic markers of platinum resistance and patient prognosis in epithelial ovarian cancer using a targeted NGS approach [23]. The patient was not included in the previous study [23] being treated with NACT-IDS. The Illumina TruSight Tumor 26-genes panel (Illumina, Inc., San Diego, CA, USA; http://www.illumina.com/products/trusight-tumor-26-gene.html) was chosen for NGS analysis, as recently reported [23]. This panel provides coverage of exon coding regions, where variation has been cataloged in the COSMIC database in oncogenes, and coverage of all 11 exons and intronic flanking regions of TP53 tumor suppressor gene. TruSight Tumor 26-genes panel gives a more comprehensive view of somatic variation in solid tumors, including lung, colon, melanoma, gastric, and ovarian cancer.

Frozen ovarian tumor specimens taken at D-LPS (pre-NACT) and during IDS (post-NACT) and a matched blood sample were analyzed retrospectively. As a WT reference sample, genomic DNA was isolated from mononuclear cells of matched peripheral blood samples collected at the time of primary surgery. Tumor samples were macrodissected and visually inspected by the pathologist to assess a minimum tumor cellularity of $70 \%$. Genomic DNA was extracted from both the tumor and blood using the EZ1 DNA Tissue Kit and EZ1 DNA Blood $350 \mu$ L Kit (Qiagen, Hilden, Germany), respectively, according to the manufacturer's instructions. DNA samples were quantified using PicoGreen Dye (Quant-iT PicoGreen dsDNA Assay Kit, Thermo Fisher Scientific, Waltham, MA, USA) on a Tecan Infinite 200 PRO reader (Tecan Trading AG, Männedorf, Switzerland) and normalized to $5 \mathrm{ng} / \mu \mathrm{L}$ for successive library preparation. DNA libraries were prepared for NGS according to the manufacturer's instructions. This panel ( $21 \mathrm{~Kb}$ size) screens 82 exons (all 11 exons of TP53 and intronic boundaries) in 26 tumor-related genes (AKT, ALK, APC, BRAF, CDH1, CTNNB1, EGFR, ERBB2, FBXW7, FGFR2, FOXL2, GNAQ, GNAS, KIT, KRAS, MAP2k1, MET, MSH6, NRAS, PDGFRA, PIK3CA, PTEN, SMAD4, SRC, STK11, TP53) across 174 amplicons (165-195 bp in size) with a 1000x minimum coverage (mean 7000x) of each amplicon. Normalized libraries were analyzed on a MiSeq platform (Illumina) using a V3 (600 cycles) sequencing flow cell with a $2 \times 121$ base pairs analysis set-up. The raw data were automatically processed and analyzed by the Illumina-Miseq system pipeline. 
VCF files were imported in Variant Studio software (Illumina Variant Studio Data Analysis Software 2.2) for variant calling and imputation. The filtering of genetic variants was performed per the following criteria: PASS filter, variant call quality equal to 100, frequency of the Alternative (Alt) allele greater or equal to $4 \%$ (TruSight Tumor panel achieves limits of detection below $5 \%$ variant allele frequency with a minimum cut-off point of $3 \%$ ), and the total number of reads passing quality filters (read depth) greater or equal to 1000x. COSMIC (https://www.cancer.sanger.ac.uk/cosmic), dbSNP (https://www.ncbi.nlm.nih.gov/snp, ClinVar databases (http://www.ncbi.nlm.nih.gov/clinvar/) and IARC TP53 Mutation Database (http://p53.iarc.fr/ProtocolsandTools.aspx), were searched to determine whether the detected variants were previously assigned ID numbers. Somatic $\mathrm{LOH}$ was determined comparing the same heterozygous and informative SNVs detected in germline vs tumor samples: an allele was considered imbalanced or relatively lost when significant changes in individual variant allele frequency (VAF), defined as absolute increases or decreases of at least 15 in the tumor, respectively, were observed as previously described [26]. We checked for statistically significant differences between pre- and post-NACT using Fisher's exact test on a $2 \times 2$ table of allele type (reference and variant) vs sample type (tumor and blood) as previously reported [27].

\subsection{Sanger Sequencing to Validate TP53 SNVs in Trans Configuration}

Sanger sequencing was performed to validate the double TP53 SNVs identified in trans configuration using genomic DNA extracted from patient's tumor tissues and blood. Variants were confirmed by at least two independent PCR amplifications and a DNA sequencing reaction on both strands. Primers used to amplify TP53 exon 4 were downloaded from the public "IARC TP53 Database" (http://p53.iarc.fr/ProtocolsandTools.aspx), TP53ex4F: 5'-TGAGGACCTGGTCCTCTGAC-3' and TP53ex4R: $5^{\prime}$-AGAGGAATCCCAAAGTTCCA-3'. Target region was PCR amplified from extracted genomic DNA; each $50 \mu \mathrm{L}$ reaction contained $1.8 \mathrm{mM} \mathrm{MgCl}_{2}, 0.25 \mathrm{mM}$ of each dNTP, $0.5 \mu \mathrm{M}$ of each primer, 20-200 ng of genomic purified DNA template, $1 \times$ PCR Buffer, $0.2 \mu \mathrm{L}$ of AmpliTaq Gold DNA polymerase (Applied Biosystems, Foster City, CA, USA), and MilliQ water. The PCR protocol was as follows: initial denaturation at $94^{\circ} \mathrm{C}$ for $2 \mathrm{~min}$, then 50 cycles of subsequent denaturation at $95^{\circ} \mathrm{C}$ for $30 \mathrm{~s}$, annealing at $57^{\circ} \mathrm{C}$ for $45 \mathrm{~s}$, and extension at $72^{\circ} \mathrm{C}$ for $1 \mathrm{~min}$, followed by the final extension step at $72{ }^{\circ} \mathrm{C}$ for $10 \mathrm{~min}$. The PCR products $(5 \mu \mathrm{L})$ were electrophoresed in $3 \%$ agarose and amplified samples cleaned up using ExoProStar (GE Healthcare). Purified reactions $(1-2 \mu \mathrm{L})$ were sequenced using the Big Dye Terminator kit (Applied Biosystems, Foster City, CA, USA) and an ABI PRISM capillary sequencer. Sequencing chromatograms were visualized with Chromas software version 2.01 (Technelysium Pty Ltd., South Brisbane, Australia) and aligned with the human TP53 reference genomic sequence.

\subsection{Immunohistochemistry}

One tumor-rich sample per case (pre-NACT at D-LPS and post-NACT at IDS), a 4- $\mu$ m-thick section from a formalin-fixed or bouin-fixed, paraffin-embedded tumor tissue block, were selected for p53 immunohistochemical analysis. One tumor-rich sample collected before therapy (pre-NACT at D-LPS) was used for BRCA1, WT-1, p16 and Ki-67 immunohistochemical analysis, respectively. Immunoperoxidase labeling was performed with the automated XT iVIEW DAB V.1 procedure on the BenchMark ULTRA IHC/ISH Staining Module, Ventana with anti-p53 (clone Bp53-11, prediluted, Ventana, Innovation Park Dr. Tucson, AZ, USA), for pre- and post-NACT tumor samples, and with anti-BRCA1 (clone MS110 prediluted, Abcam, Discovery Drive, CB, UK), anti-p16 (clone E6H4, ready to use, Ventana), anti-Wt1 (clone 6F-H2 ready to use, Ventana), anti-Ki-67 (clone 30-9 ready to use, Ventana), for only the pre-NACT tumor sample. Sections were incubated with: anti-p53 for $16 \mathrm{~min}$ at $37^{\circ} \mathrm{C}$, anti-BRCA1 for $36 \mathrm{~min}$ at room temperature, anti-p16 for $16 \mathrm{~min}$ at $36^{\circ} \mathrm{C}$, anti-WT1 for $40 \mathrm{~min}$ at $37^{\circ} \mathrm{C}$, and anti-Ki-67 for $32 \mathrm{~min}$ at $42{ }^{\circ} \mathrm{C}$. Antigen retrieval was carried out with CC1 (Ventana) for all primary antibodies. Stainings were detected using the I-View DAB detection system. All slides were reviewed by the pathologist (VC), who was blinded to molecular data. Nuclear staining was considered a positive reaction. The extent of nuclear or cytoplasmic staining was estimated to the 
nearest $5 \%$ level of positive tumor cells, reporting the actual percentage for each case. The intensity of staining was recorded as - (Absent); -/+ (Weak); + (Moderate); ++ (High); or +++ (Very High).

\section{Results}

\subsection{Case History}

A 63-year-old patient was referred to CRO Aviano-National Cancer Institute for suspected advanced ovarian carcinoma with presence of abundant abdominal and pleural ascites (clinical stage IV). She denied familial history of ovarian and/or breast cancer. In February 2005, through an explorative D-LPS, the patient was deemed unresectable because of diffuse carcinomatosis with deeply infiltrating nodules, thus, undergoing only aspiration of hemorrhagic ascites $(\sim 2300 \mathrm{~mL})$, right ovariectomy, partial omentectomy, and the collection of multiple biopsies. Then, NACT was recommended. The pathological diagnosis was poor differentiated (tumor grade G3) infiltrating ovarian adenocarcinoma ( $\mathrm{pT} 3 \mathrm{cNx}$ ) of the serous sub-type, and the patient was assigned to FIGO stage IV for the presence of distal pleural effusion. CA-125 level (normal value is $<35 \mathrm{U} / \mathrm{mL}$ ) before chemotherapy was $608.0 \mathrm{U} / \mathrm{mL}$ (Figure 1).

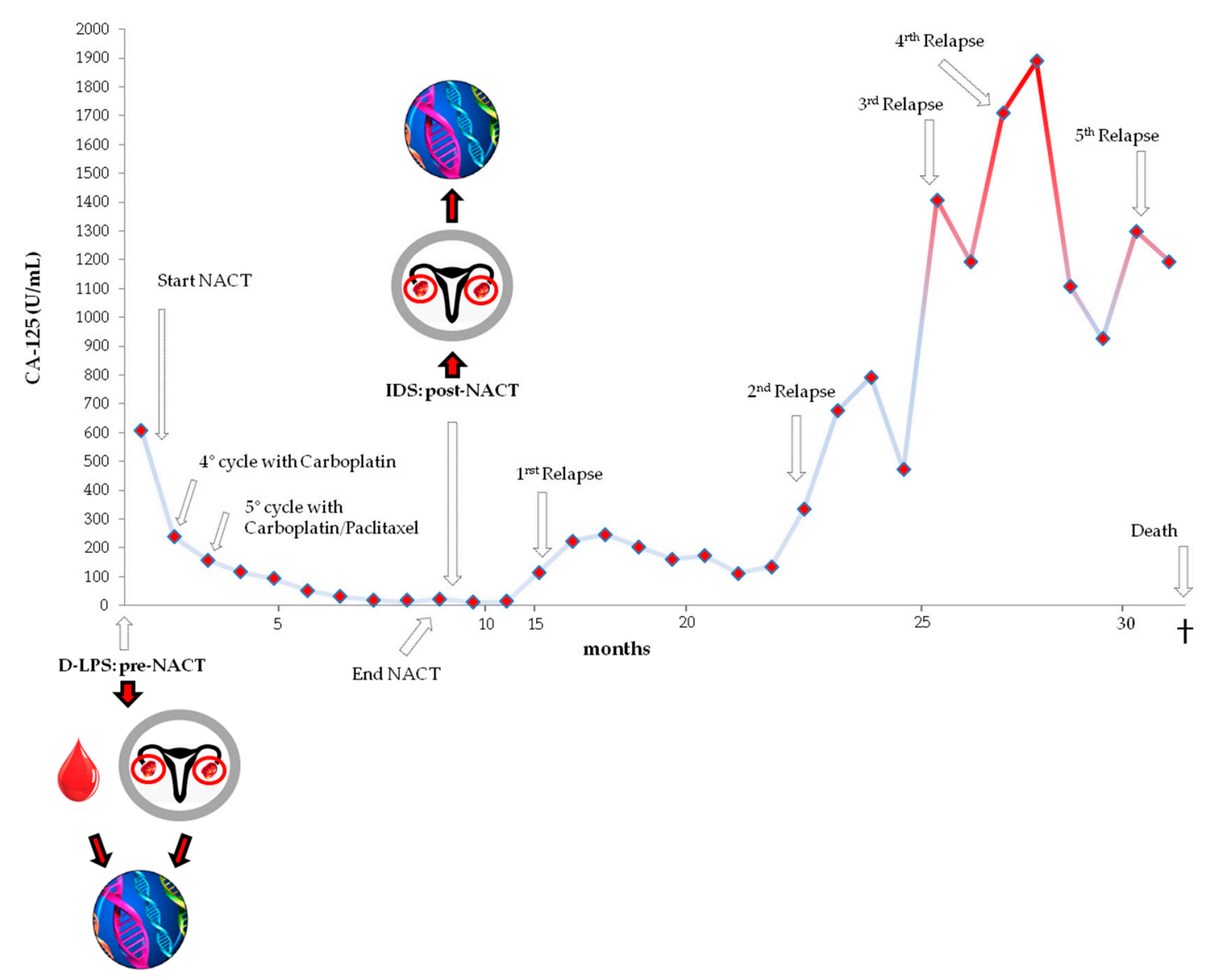

Figure 1. Graphical representation of CA-125 levels across the disease/treatment course of the patient diagnosed with HGSOC. The two time points, at D-LPS (pre-NACT) and at IDS (post-NACT), in which blood and ovarian tumor tissue samples were collected, are highlighted. CA-125: cancer antigen 125; D-LPS: diagnostic laparoscopy; NACT: neo-adjuvant chemotherapy; IDS: interval debulking surgery; HGSOC: high-grade serous ovarian cancer.

The patient underwent four cycles of NACT (carboplatin alone, area under curve (AUC) 5 with one intrapleural administration of Bleomycin before the second cycle of NACT), that was then prolonged with six additional cycles of carboplatin/paclitaxel (carboplatin AUC 5 and paclitaxel $255 \mathrm{mg} / \mathrm{m}^{2}$ ), due 
to poor clinical response to platinum based on Response Evaluation Criteria In Solid Tumors (RECIST) criteria of computed tomography (CT) scan and CA-125 level, still high (239.0 U/mL). After a total of ten cycles, the chemotherapeutic response was only partial with normalization of CA-125 level but with positron emission tomography (PET)/CT scan still revealing multiple peritoneal nodules and pleural effusion. According to the National Cancer Institute-Common Toxicity Criteria version 2.0 (NCI-CTC v. 2.0), the patient experienced grade G4 neutropenia after the 5th, 7th, and 9th cycle, G1 and G2 alopecia after the 5th and the 6th cycle respectively, and G1 fatigue after the 9th cycle. Neurotoxicity was not reported. Surgery (IDS) was then attempted with hysterectomy, left ovariectomy, appendectomy, and partial intestinal resection for presence of peritoneal and mesenteric nodules $(\mathrm{RD}<0.5 \mathrm{~cm})$. Upon the histological assessment of the surgical specimens, the ovarian cancer was classified as poor differentiated/undifferentiated (G3/4), FIGO stage IIIC (ypT3cNx) serous ovarian cancer. After IDS three additional cycles with only carboplatin were administered to the patient. Disease progression was detected in peritoneum with increased dimension of peritoneal nodules (revealed by PET/CT) and also evidenced by the biochemical increase in CA-125 marker $(116.0 \mathrm{U} / \mathrm{mL})$, just less than five months from the last carboplatin administration. The patient was defined as platinum-resistant (PFI 4.4 months) and TTR was 7.5 months. The patient received a second-line chemotherapy with carboplatin/PDL (Pegylated liposomal Doxorubicin) but after the eighth cycle the treatment was stopped for disease progression (peritoneal dissemination and CA-125 increase, $334.3 \mathrm{U} / \mathrm{mL}$ ). The patient was then treated with Capecitabine but after three cycles the chemotherapy was interrupted for disease progression (peritoneal dissemination and CA-125 increase, $1407.0 \mathrm{U} / \mathrm{mL}$ ). Then, hormonal therapy with Letrozole was given to the patient for about one month until the detection of progression (peritoneal dissemination and CA-125 increase, $1711.0 \mathrm{U} / \mathrm{mL}$ ). Finally, the patient received three cycles of Gemcitabine which was stopped (disease progression) and substituted with palliative care until death. The OS was 30.7 months.

\subsection{Molecular Analysis by NGS}

NGS was performed on frozen ovarian cancer tissues of the patient collected at the D-LPS (pre-NACT) and during IDS (post-NACT). A blood sample collected at D-LPS was used as a matched control/reference. Analysis of the 26 tumor-related genes on the targeted NGS panel (BRCA1/2 were not included in the commercial panel) was carried out using Variant Studio after filtering genetic variants using the following criteria: PASS filter, variant call quality $=100$, VAF of the Alt allele $\geq 4 \%$, and total number of reads passing quality filters (read depth) $\geq 1000 x$. Among the 26 tumor-related genes, one somatic SNV was detected only in TP53. Another germline SNV was detected in tumor samples and in the matched normal/blood sample. VAF was calculated in each sample as the ratio of the number of sequence reads documenting the alternative allele versus all sequence reads aligned to that position. SNVs were automatically annotated against the human TP53 reference genomic sequence NC_000017.10 (chr 17: 7,571,720-7,590,868) corresponding to isoform NM_000546.5 (Table 1).

Table 1. TP53 SNVs identified by NGS in a patient with HGSOC treated with NACT.

\begin{tabular}{|c|c|c|c|c|c|c|c|c|c|c|}
\hline $\begin{array}{c}\text { Blood } \\
\text { (Germline) }\end{array}$ & $\begin{array}{c}\text { Tumor } \\
\text { Pre-NACT }\end{array}$ & $\begin{array}{c}\text { Tumor } \\
\text { Post-NACT }\end{array}$ & $\begin{array}{c}\text { TP53 Gene } \\
\text { Region }\end{array}$ & $\begin{array}{c}\text { Genomic } \\
\text { Coordinate * }\end{array}$ & $\begin{array}{l}\text { cDNA } \\
\text { Change }\end{array}$ & $\begin{array}{l}\text { Ref }_{-} \\
\text {seq }\end{array}$ & $\begin{array}{l}\text { Alt } \\
\text { seq }\end{array}$ & $\begin{array}{l}\text { Variant } \\
\text { Type }\end{array}$ & $\begin{array}{c}\text { AA } \\
\text { Change }\end{array}$ & $\begin{array}{c}\text { Predicted } \\
\text { Protein } \\
\text { Product }\end{array}$ \\
\hline No & Yes & Yes & IVS4 & $17: 7,579,311$ & c. $375+1 G>A$ & G & A & SD & p.Gly59Valfs*23 & Truncated \\
\hline
\end{tabular}

* Reference build is GRC37/h19. SNV: single nucleotide variant; NGS: next-generation sequencing; HGSOC: high-grade serous ovarian cancer; VAF: variant allele frequency; Ref: reference allele; Alt: alternative allele; AA: amino acid; SD: splice donor; NACT: neo-adjuvant chemotherapy; IVS4: intervening sequence 4, i.e., intron sequence 4; Gly: glycine; Val: valine; fs: frameshift; Pro: proline; Arg: arginine.

The somatic mutation TP53 c.375+1G>A (i.e., TP53 IVS4+1G>A) was detected in pre- and post-NACT tumor samples from HGSOC. This mutation affects the first nucleotide downstream exon 4 that belongs to a canonic splice donor (SD) site, consisting of two conserved GT nucleotides. 
This variant has been already reported both in the International Agency for Research on Cancer (IARC) TP53 Mutation Database (Database R19, released August 2018) [28] and in COSMIC (mutation ID: COSM45304), while it has not been annotated in dbSNP and ClinVar databases. Presence of TP53 c.375+1G > A mutation induces aberrant splicing with activation of a cryptic splice donor site in codon 59 in exon 4 [29], with a consequent frameshift and formation of a premature stop codon, as demonstrated by the functional analysis FASAY, which uses mRNA as starting material $[29,30]$. A germline single nucleotide polymorphism (SNP) in exon 4, the TP53 c.215C $>\mathrm{G}$, was identified in the blood and also in both HGSOC samples. This SNP codifies for a nonsynonymous/missense amino-acid (AA) change from proline (Pro) to arginine (Arg) in codon 72 (p.Pro72Arg), and it has been annotated in COSMIC (COSM250061), dbSNP (rs1042522) and ClinVar (Variation ID 12351) databases. Minor allele frequency (MAF) for SNP rs1042522 is 28.5\% (G) in Eur (european) population in Ensemble database (http://grch37.ensembl.org/Homo_sapiens/Info/Index) and 34.0\% (G) in ExAC Browser (http://exac.broadinstitute.org/). MAFs for TP53 c.375+1G $>$ A are unreported since this mutation has been described only at the somatic level. Presence of genetic polymorphism in codon 72 leads to the alteration of protein structure and decreases apoptosis. In particular, Arg at position 72 was shown to enhance p53 related apoptotic potential, rather than Pro at the same position [31-35]. SNP rs1042522 has been associated with a possible impact in drug response for clinical significance according to ClinVar. In the tumor samples collected at D-LPS and IDS, the SNVs identified in TP53 are within 160 nucleotides of genomic interval from each other and are both C: $G>T$ : A transitions. VAF for mutation TP53 c.375+1G >A in pre-NACT chemo-naïve tumor sample was $50.13 \%$, while in post-NACT tumor sample at IDS was $94.33 \%$. VAF for polymorphism TP53 c. $215 \mathrm{C}>\mathrm{G}$ was $27.71 \%$ and $4.21 \%$, in pre-NACT and in post-NACT tumor samples respectively, while in blood samples at the germline level was $57.3 \%$ (Figure 2).

NGS results configured that the double SNVs identified, one in exon 4 (TP53 c.215C >G) and one in the flanking intronic region (TP53 c.375+1G>A) of the same exon, were in trans configuration [33] in the HGSOC analyzed. 


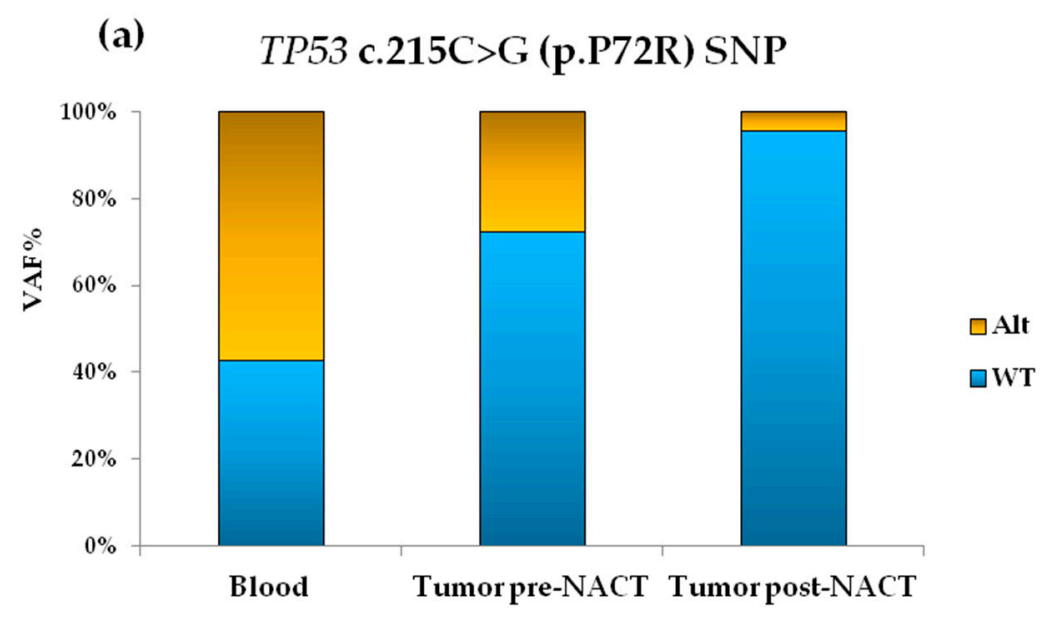

(b)

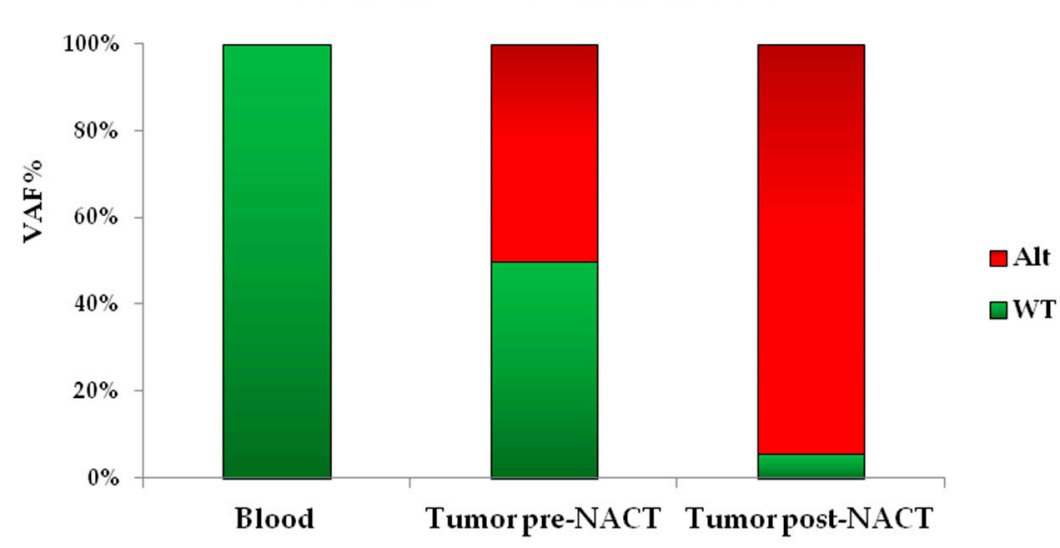

Figure 2. Graphic representation of VAF observed for SNVs in TP53 identified by NGS in blood (reference) and tumor tissue samples, collected at pre-therapy (pre-NACT) at the D-LPS and post-therapy (post-NACT) at the IDS, from a patient with HGSOC. (a) Bar-graph shows VAF decrease in pre- (27.71\%) and post-NACT (4.21\%) tumor samples, indicating prevalence of the $\mathrm{WT}$ " $\mathrm{C}^{\text {" }}$ allele (blue bar) compared to the Alt "G" allele (yellow bar) at IDS for the TP53 c.215C>G (p.P72R) SNP, present in heterozygosis $(57.31 \%)$ in the blood reference sample; (b) Bar-graph shows VAF increase in pre- $(50.13 \%)$ and post-NACT (94.33\%) tumor samples highlighting prevalence of the Alt " $A$ " allele (red bar) compared to the WT "G" allele (green bar) in the HGSOC tissue sample collected at IDS of TP53 c.375+1G>A mutation, absent in the blood reference sample (only WT " $G$ " allele, green bar). VAF: variant allele frequency; NACT: neo-adjuvant chemotherapy; HGSOC: high-grade serous ovarian cancer; D-LPS: diagnostic laparoscopy; IDS: interval debulking surgery; Alt: alternative; WT: wild-type.

\subsection{Validation of NGS Results by Sanger Sequencing}

To confirm the particular trans configuration of double SNVs identified in TP53, standard Sanger sequencing was performed using the identical genomic DNA isolated from the tumor samples and blood. Sanger sequencing confirmed NGS results and the trans configuration of double SNVs identified in TP53 (Figure 3). 
(a) Blood

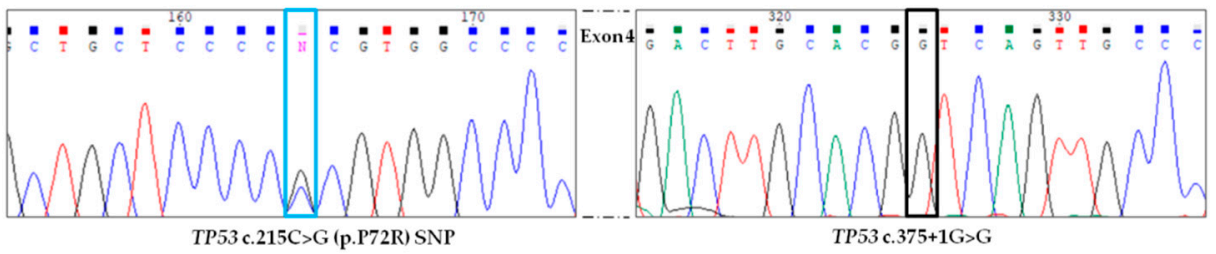

(b) Tumor pre-NACT

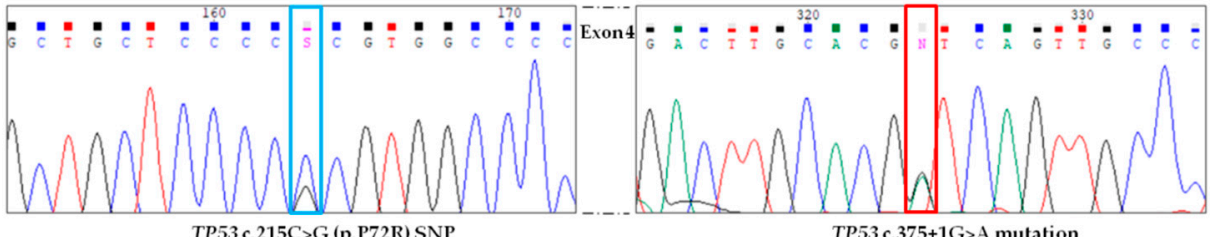

TP53c.215C>G (p.P72R) SNP

TP53 c. $375+1 \mathrm{G}>$ A mutation

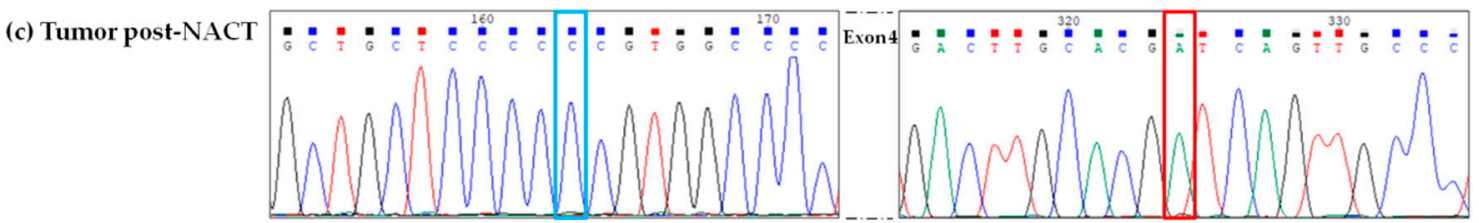

TP53 c.215C >G (p.P72R) SNP

TP53 c. 375+1G $>$ A mutation

Figure 3. Confirmatory Sanger electropherograms of TP53 variants identified by NGS in a patient with HGSOC, in tumor samples collected at D-LPS (pre-NACT) and at IDS (post-NACT), and in reference (germline) blood sample. (a) Sequencing result in the reference blood sample showing polymorphism TP53 c.215C > G in heterozygosis (blue box) and the WT " $G$ " allele at the TP53 position c.375+1G>G (black box); (b) Sequencing result in the tumor sample collected at the D-LPS (pre-NACT) of the polymorphism TP53 c.215C>G (increased peak for WT "C" allele, blue box) and mutation TP53 c.375+1G > A (heterozygosis, red box), in trans configuration; (c) Sequencing result in the tumor sample collected at the IDS (post-NACT) of the polymorphism TP53 c.215C > G indicating homozygosis of the WT " $C$ " allele or loss of the Alt " $G$ " allele (blue box) and mutation TP53 c.375+1G>A indicating homozygosis of the Alt " $A$ " mutated allele or loss of the WT " $G$ " allele (red box), in trans configuration. HGSOC: high-grade serous ovarian cancer; D-LPS: diagnostic laparoscopy; IDS: interval debulking surgery; NACT: neo-adjuvant chemotherapy; SNP: single nucleotide polymorphism; NGS: next-generation sequencing; WT: wild-type.

Further, peaks visualized in the chromatograms from Sanger sequencing were concordant with VAFs obtained with NGS approach. However, the standard sequencing approach could not discriminate allelic imbalance from a homozygous nucleotide change. In the reference (germline) blood sample, the presence of TP53 c.215C > G polymorphism in heterozygosis and absence of the mutation TP53 c.375+1G $>$ A was evidenced (Figure 3a). In the pre-NACT chemo-naïve sample, the intronic alteration flanking exon 4 in heterozygous state was confirmed, as well the presence of polymorphism TP53 c.215C > G with a diminished peak related to the " $G$ " variant allele (Figure 3b). In post-NACT tumor sample, both SNVs identified showed homozygosis of Alt/mutant " $A$ " allele (or suspected loss of the of the WT " $G$ ") and of the WT " $C$ " allele (or suspected loss of the Alt/mutant " $G$ " allele), for the TP53 c. $375+1 G>A$ and TP53 c. $215 C>G$ variants, respectively (Figure 3c).

\subsection{Somatic $\mathrm{LOH}$}

SNVs may be noninformative homozygous for either a reference or a variant SNV, or heterozygous and informative. In tumor cells, either the reference or variant heterozygous SNV may be entirely lost or amplified. To determine $\mathrm{LOH}$, the difference and the absolute values between the tumor samples VAF and the matched normal (blood) sample VAF of the only one germline heterozygous and informative SNV identified (TP53 c.215C >G), were calculated. The absolute differences between VAFs detected for TP53 c.215C > G SNP in pre-NACT tumor sample, post-NACT tumor sample and blood were 29.6 and 53.1, respectively ( $p<0.0001$, for both). The germline heterozygous SNP showed 
significant changes in VAF, indicating allelic imbalance in the HGSOC after completion of NACT, as well in the primary untreated/chemo-naïve tumor. However, in the pre-NACT/chemo-naïve tumor sample, the somatic SNV was in heterozygosis (VAF $=50.13 \%$ ) as evidenced also by the less sensitive Sanger sequencing (Figure 3b), indicating that either the sample was comprised of tumor and normal cells or clonal heterogeneity was present in the untreated tumor. SNVs identified in trans configuration were strongly affected by somatic LOH in the post-NACT tumor sample at IDS, with clone expansion resulting in the loss of the reference allele for TP53 c.375+1G $>$ A mutation and loss of the Alt/variant allele of TP53 c.215C > G SNP (Figure 4).
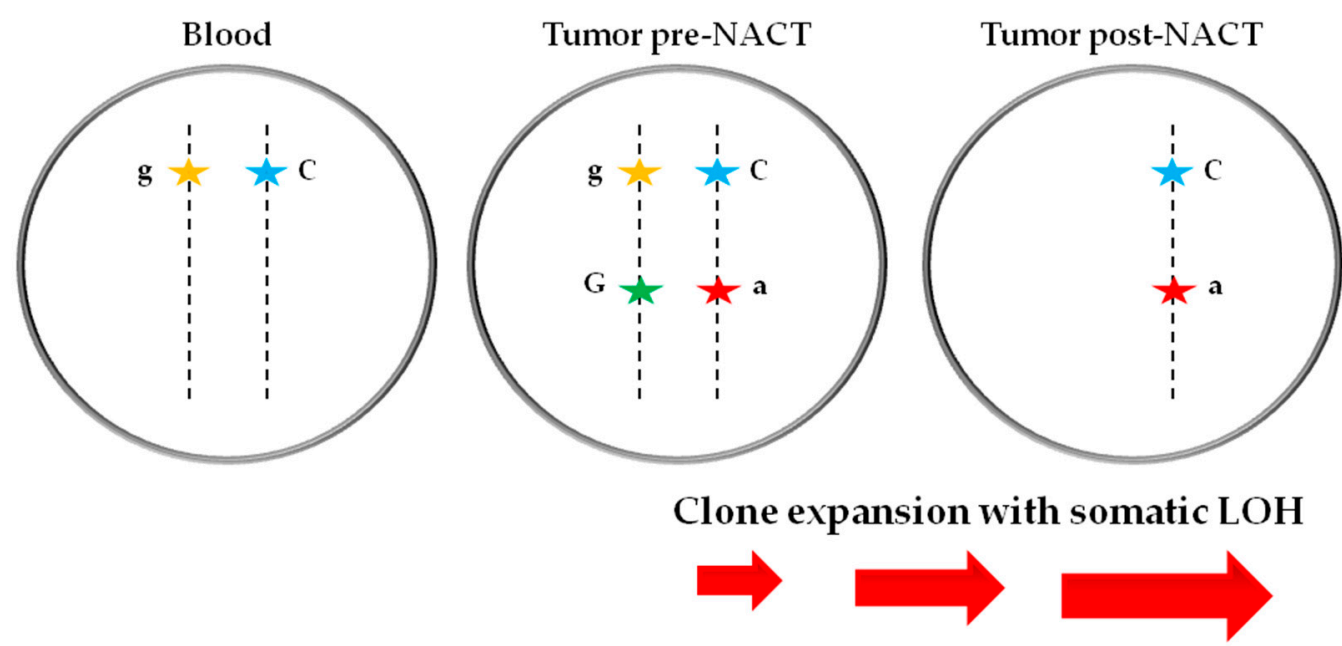

Figure 4. Representative model of double TP53 SNVs in trans configuration identified in the pre-NACT/chemo-naïve HGSOC sample at D-LPS, with clone expansion and somatic LOH in the post-NACT tumor sample collected at IDS. In normal cells (Blood) at the germline level, only the TP53 SNP c.215C > G was detected; in the chemo-naïve/untreated (Tumor pre-NACT) sample, the splice mutation TP53 c.375+1G>A was present with the minor Alt allele in trans to the WT allele of polymorphism TP53 c. $215 \mathrm{C}>\mathrm{G}$; in the post-therapy/treated (Tumor post-NACT) sample, the complete clone expansion with somatic $\mathrm{LOH}$ was observed with loss of the minor Alt allele for TP53 c.215C $>\mathrm{G}$ SNP and loss of the WT allele for TP53 c.375+1G >A mutation. Alt allele of the mutation TP53 c.375+1G $>\mathrm{A}$ is represented in red, the reference WT in green; Alt allele of the polymorphism TP53 c. $215 \mathrm{C}>\mathrm{G}$ is colored in orange, the reference WT allele in blue; the minor Alt alleles are in lower-case letters, the WT alleles are in upper-case letters. VAF: variant allele frequency; NACT: neo-adjuvant chemotherapy; HGSOC: high-grade serous ovarian cancer; D-LPS: diagnostic laparoscopy; IDS: interval debulking surgery; Alt: alternative; WT: wild-type; LOH: loss of heterozygosity.

\subsection{Immunohistochemical Evaluation of p53 Expression in HGSOC Tumor Samples}

HGSOC showed papillary, glandular, and solid architecture with psammoma bodies in D-LPS/pre-NACT (Figure 5a-c) and micropapillary morphology in IDS/post-NACT (Figure 5g-i) tumor tissue samples. 
H\&E

Tumor pre-NACT

p53

Tumor pre-NACT

H\&E

Tumor post-NACT

p53

Tumor post-NACT
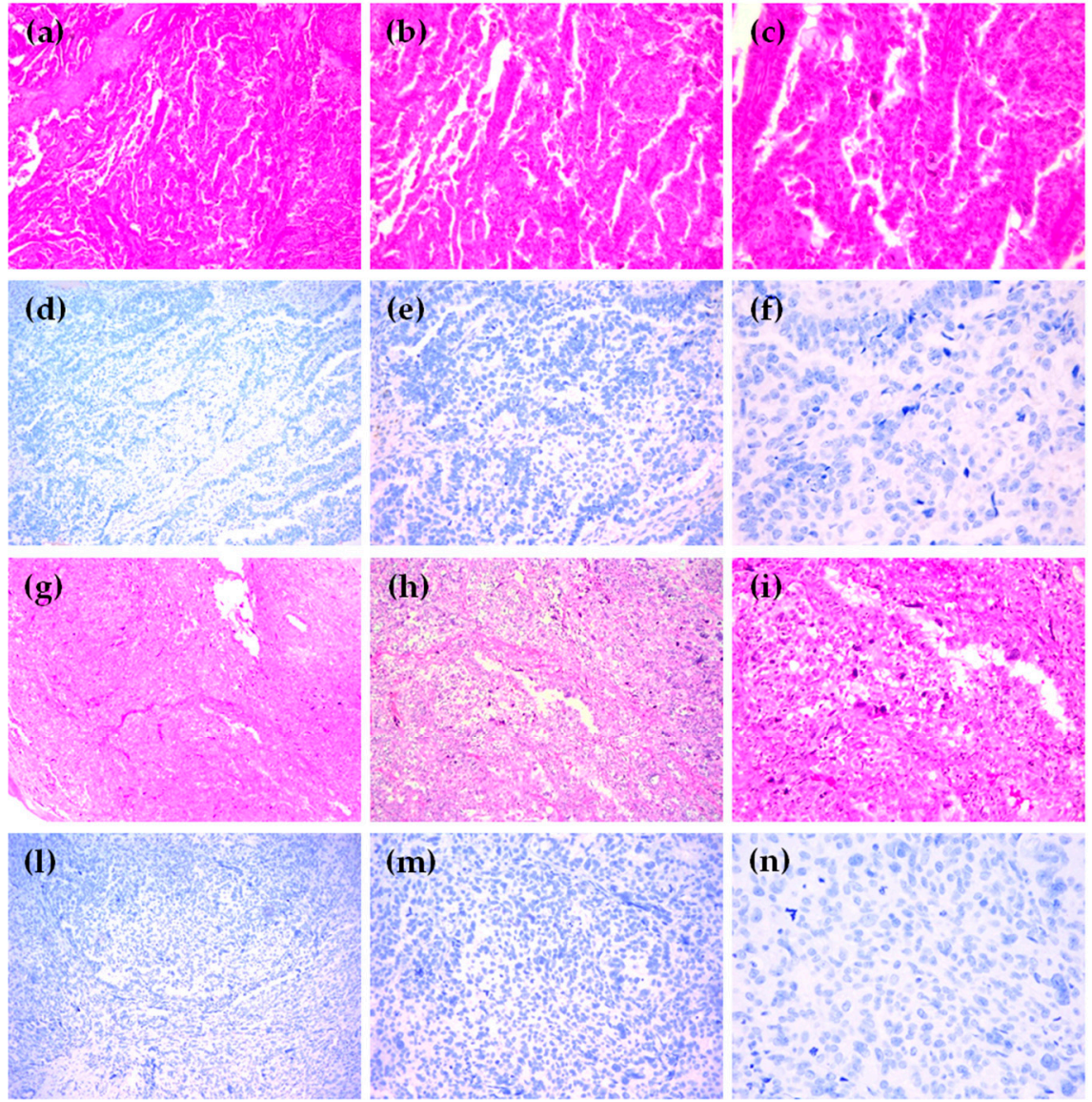

\section{(m)}

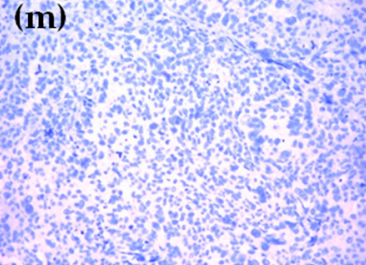

(n)

Figure 5. Hematoxilin and eosin (H\&E, $5 \times, 10 \times$ and $20 \times$ magnification) of HGSOC sections collected at D-LPS (pre-NACT) and at IDS (post-NACT) and immunohistochemical staining for p53 (10 $\times, 20 \times$ and 40× magnification). (a-c) H\&E of bouin-fixed, paraffin-embedded tumor tissue collected at D-LPS showing the serous ovarian carcinoma architecture of HGSOC case with somatic mutation in TP53 (a: 5x; b: 10x; c: 20x); (d-f) Absent (-) nuclear p53 expression on tumor tissue collected at D-LPS of HGSOC case with somatic mutation in TP53 (d: 10x; e: 20x; f: 40×); (g-i) H\&E of formalin-fixed, paraffin-embedded tumor tissue collected at IDS showing the serous ovarian carcinoma architecture of HGSOC case with somatic mutation in TP53 (g: 5x; h: 10x; i: 20x); (1-n) Absent (-) nuclear p53 expression on tumor tissue collected at IDS of HGSOC case with somatic mutation in TP53 (1: 10x; m: 20×; n: 40×). No cytoplasmic staining was observed. NACT: neo-adjuvant chemotherapy; HGSOC: high-grade serous ovarian cancer; D-LPS: diagnostic laparoscopy; IDS: interval debulking surgery.

Severe nuclear atypia (multinucleated tumor giant cells) was observed in both pre- and post-NACT samples, in particular in chemo-naïve sample (Figure 5c). Immunohistochemical staining of p53 was completely negative (-) in tumor cell nuclei and cytoplasm, in both D-LPS/pre-NACT (Figure 5d-f) and IDS/post-NACT (Figure 5l-n) tissue samples. In the primary chemo-naïve tumor, BRCA1, p16, WT1, and Ki-67 markers were also evaluated by immunohistochemistry analysis.

BRCA1 expression was positive in about $95 \%$ of tumor cells with a weak $(+)$ nuclear staining intensity (Figure 6a,b). 


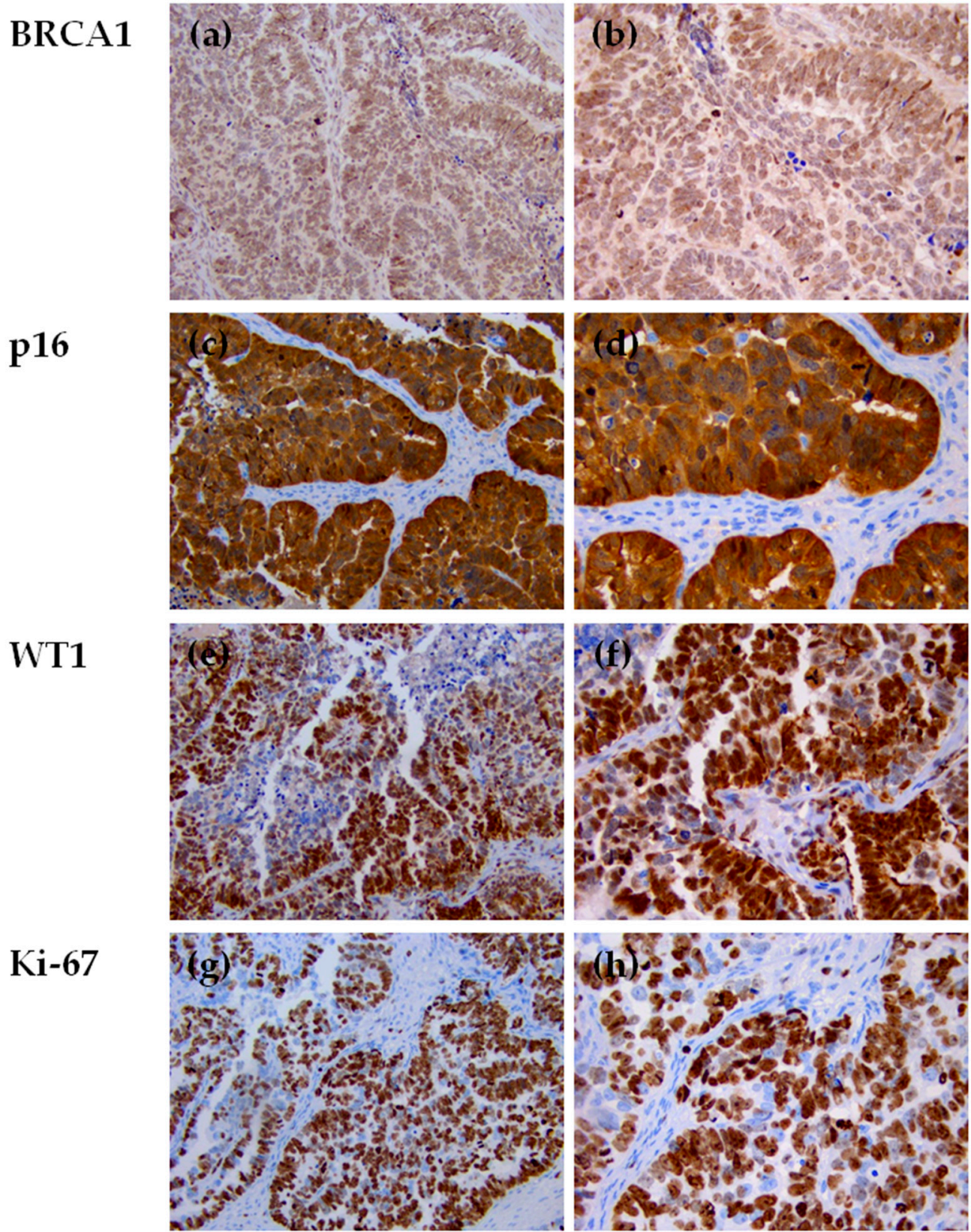

Figure 6. Immunohistochemical staining for BRCA1, p16, WT1, and Ki-67 of HGSOC sections collected at D-LPS from a patient diagnosed of HGSOC, before NACT ( $20 \times$ and $40 \times$ magnification). (a,b) Positive nuclear staining for BRCA1 observed in $~ 95 \%$ of tumor cells (a: 20×, b: 40×); (c,d) Diffuse nuclear and cytoplasmic staining of p16 exhibited by $~ 95 \%$ of tumor cells (c: $20 \times$, d: $40 \times)$; (e,f) Nuclear and cytoplasmic staining for WT1 observed in $\sim 70 \%$ of tumor cells (e: $20 \times, \mathbf{f :} 40 \times)$;(g,h) Diffuse nuclear staining of Ki-67 observed in 95\% of tumor cells (g: 20×, h: 40×). NACT: neo-adjuvant chemotherapy; HGSOC: high-grade serous ovarian cancer; D-LPS: diagnostic laparoscopy; BRCA1: BRCA1 DNA repair associated; WT1: Wilms tumor 1.

Diffuse nuclear and cytoplasmic staining was observed for p16 (i.e., cyclin dependent kinase inhibitor $2 \mathrm{~A}$ (CDKN2A) protein) in $\sim 95 \%$ of tumor cells with $\sim 20 \%$ of cells with weak $(+), \sim 55 \%$ with moderate $(++)$ and $\sim 20 \%$ with high $(+++)$ intensity (Figure $6 \mathrm{c}, \mathrm{d}$ ). Nuclear and cytoplasmic staining for WT1 protein was exhibited in $\sim 70 \%$ of tumor cells: weak $(+)$ intensity was observed in the cytoplasm while nuclear staining showed $\sim 20 \%$ of cells with weak $(+), \sim 20 \%$ with moderate $(++)$ and $\sim 30 \%$ with high $(+++)$ intensity (Figure 6e,f). The percentage of Ki-67 positive tumor nuclei (Ki-67 proliferation 
index) [34] was $\sim 95 \%$ with $\sim 20 \%$ of cells with weak $(+), \sim 40 \%$ with moderate $(++)$ and $\sim 35 \%$ with high $(++)$ staining intensity (Figure $6 \mathrm{~g}, \mathrm{~h}$ ).

\section{Discussion}

In advanced ovarian cancer, the benefit from NACT based on platinum and paclitaxel followed by IDS, is still a matter of debate. The possibility that NACT may induce chemoresistance before and after IDS has been poorly investigated $[6,8,15,16]$. By means of targeted NGS, matched pre-and post-neoadjuvant platinum/paclitaxel-based chemotherapy tumor samples, as well the normal/blood reference sample, from a patient with stage IV HGSOC treated with NACT-IDS, were analyzed. Expansion of the TP53 c.375+1G > A driver mutant clone with somatic LOH and loss of the 72Arg allele of p53 after NACT were observed, suggesting a possible mechanism of chemoresistance in HGSOC. The patient had a very aggressive clinical course of ovarian disease, which underlined the aggressive tumor phenotype. Immunohistochemical analysis indicated that tumor cells were highly proliferative (Ki-67) and exhibited a typical phenotype of HGSOC with the expression of p16 and WT1 markers. Expression of the Ki-67 marker is strongly associated with cell proliferation and is commonly used in routine diagnostic pathology [36], also to distinguish HGSOC from low-grade serous ovarian cancer (LGSOC) [37]. WT1 is not expressed in the epithelium of healthy fallopian tubes, which is assumed to be the site of origin of most HGSOC [38], and it is a recognized marker of serous differentiation, useful to distinguish serous tumors (both high-grade from low-grade) from other tumor types [39]. Diffuse p16 staining supports a diagnosis of HGSOC, especially when p53 expression is uncertain [40]. The prognostic value of WT1 expression marker has been also investigated in HGSOC, but with opposite effects [38,41]. The analyzed chemo-naïve tumor showed WT1 positive, absent p53 and diffuse p16 immunostaining, which is concordant with HGSOC phenotype; conversely, LGSOC are characterized by positive expression of WT1 and by WT pattern for p53 and patchy for p16 [39]. The patient was refractory to treatment after four cycles of platinum, and despite CA-125 normalization levels, she had a poor pathological response at the end of the following six cycles with the addition of paclitaxel. No increase in the tumor mutational or neoantigen load was found when comparing tumor samples prospectively collected before and after NACT. This result is in agreement with that reported for other studies in urothelial [42] and in bladder [43] primary carcinomas, in which matched pre- and post-neoadjuvant cisplatin-based chemotherapy tumor samples were analyzed by NGS. The somatic mutation identified in TP53 was unique in both matched pre-and post-therapy tumor samples, similarly to recent previous research in HGSOC [22,44]. Direct induction of TP53 mutations by DNA-damaging chemotherapy with alkylating agents is a possible recognized event [45]. The first relevant finding in this study is that the somatic mutation identified in TP53 was not a consequence of DNA damaging drugs because it arose before the start of chemotherapy treatment. This result is also suggestive that the driver splice mutation detected conferred a selective advantage and in vivo chemotherapy resistance, being the only one somatic variant identified in post-therapy sample, according to the 26 cancer-genes panel used. Indeed, the selection of pre-existing mutated clones by chemotherapy was reported as the predominant mechanism of TP53 mutation expansion [46,47]. However, the association of TP53 mutations with chemoresistance in cancer [48] and in HGSOC $[23,49]$ is still controversial. The splice variant TP53 c.375+1G $>$ A, affecting a consensus SD site, is an infrequent somatic mutation (among those reported for TP53) already described in sporadic breast cancer non-carriers in BRCA1 mutations [50], in situ skin cancer lesions [51], bladder cancer [52], esophageal squamous cell carcinoma [29,53], ovarian cancer [54], HGSOC [55], and in other type of malignancies as currently reported in COSMIC database. Other somatic mutations affecting the consensus (TP53 c.375+1G > T, TP53 c.375+2T>A) and non-consensus (TP53 c.375+5G>A) SD site in exon 4 flanking region of TP53 have been already reported in different malignancies, including HGSOC, and they were associated with similar altered splicing of TP53 c.375+1G $>$ A, with additional aberrations (i.e., insertion of whole intron 4 and frameshift, insertion of 109 nucleotides of intron 4 and frameshift) [30]. We also evaluated, with the same targeted NGS panel used in this study, the entire 
coding region of TP53 and the related intronic boundaries in 79 chemo-naïve advanced (III-IV stage, tumor grade G2-3) ovarian tumors, finding splice site mutations only in the sub-group of HGSOCs, in six patients (11.5\%) [23]. Intriguingly, three out of six patients had somatic splice mutations affecting directly (TP53 c.375+1G > T and TP53 c.375+2T >A), and non-directly (TP53 c.375+5G >A), the SD site in exon 4 flanking region of TP53 [23]. Moreover, all six patients were also carriers of the polymorphism TP53 p.Pro72Arg and the one mutated for TP53 c.375+5G $>$ A had also a concurrent mutation in KRAS (KRAS p.G12C), contributing to define a poor prognostic signature in patients with HGSOC [23]. These patients with somatic splice mutations in intron 4 of TP53 were also characterized by a poor prognosis (median PFI $<4$ months, median TTR $<12$ months, median OS $<27$ months).

The amount of mutations in TP53 implicated in splice junctions that interfere with correct protein translation, by introducing frameshift or aberrant spicing, has been probably underestimated in many studies, due to the restriction of genetic analyses in coding regions and in the DNA binding domain (DBD) of p53. Many rare genomic alterations, such as those affecting the splice site regions, have been unveiled by the application of NGS, thus expanding the potential repertoire for the use of target therapies and personalized therapy [24]. Aberrant splicing events are a common phenomenon in tumorigenesis described in each of the accepted hallmarks of cancer, particularly in apoptosis and metastasis formation, even if, their biological functions are still not completely clarified [56]. Splice site mutations when categorized as disruptive or predicting a non-functional p53 protein together with nonsense and frameshift variants [57] have shown a poor prognostic value in squamous head and neck cancer [58] and in a large cohort of patients with breast cancer [59]. Moreover, disruptive TP53 mutations showed significant radioresistance with a failure of senescence induction, compared to nondisruptive/missense mutations [58]. The downstream effects of splice mutations on target gene expression and pathway activation are still poorly investigated. Nonetheless, some truncated p53 isoforms associated to a p53-null state named p53 $\psi$, derived from splice acceptor (SA) site mutations (TP53 c.673-2A>T/C/G; TP53 c.673-1G>T/C/A), are capable of reprogramming cells inducing the acquisition of pro-metastatic features $[60,61]$. A recent study evaluated the amount of TP53 splice mutations through the sequencing of exons 2-11 and ten intronic flanking nucleotides for each exon, in a series of 401 primary non-metastatic colorectal cancers (CRCs): the number of these type of mutations detected was three-fold higher than reported in major databases [62]. Furthermore, splice mutations were found to be significantly associated with worse relapse-free survival, defining a high-risk group for stage II CRC [62].

The TP53 c.375+1G>A mutation affects directly p53 splicing [29] producing a short truncated protein without most of known coding sequence, including the functional DBD and the oligomerization domain (OD), which contains also the localization and exportation signals for nuclear importation and exportation of p53, respectively [25]. Therefore, unsurprisingly, expression of p53 in both pre- and post-NACT tumor samples, that showed completely negative immunostaining, indicating abnormal p53 expression [39], and loss of the p53-WT functions, was not observed. Notably, negative p53 tumor staining was concordantly reported in two patients with primary/chemo-naïve HGSOC, one with the same somatic mutation TP53 c. $375+1 \mathrm{G}>\mathrm{A}$, and the other mutated for TP53 c.375+2T>G, affecting the analogous SD site [55].

Codon 72 of p53 human protein is localized in the proline-rich domain (residues 58-101), a hydrophobic region characterized by five copies of sequence PXXP, whereas P is Pro and X any AA residue [63]. This motif forms a left-handed polyPro type II helix which creates a binding site for src homology-3 (SH3) domain [64]. The relevance of the PXXP sequence is supported by the fact that this motif has been found in all the proteins known to bind directly to SH3 domains [65]. The PXXP motif, due to its conservation in different species, is considered a discrete signal module playing a critical role in the transmission of antiproliferative signals [65]. Moreover, p53 mutants lacking 62-91 residues demonstrated that the proline-rich domain is required to induce apoptosis and cell death in presence of compounds used in cancer treatment causing DNA damage, which sensitize cells to p53 dependent apoptosis [63]. When cells without the five PXXP domains were treated with different chemotherapeutic 
drugs (camptothecin, colchicines, daunorubicin, etoposide, 5-fluorouracil), they failed to undergo apoptosis [63]. Genetic polymorphism in codon 72 with AA exchange from Pro to Arg, leads to the loss of one of the five PXXP repeats with alteration of p53 protein structure and p53-dependent apoptosis induction $[31,32,66]$. In particular, Arg at position 72 was shown to enhance the apoptotic potential more than Pro at the same position, leading mutated cells with 72Arg allele, to acquire an increased sensitivity with a more vigorous apoptotic response after chemotherapy treatment $[35,66,67]$, partially through targeting p53 to the mitochondria [31]. Presence of the 72Pro allele has been associated with poor survival and prognosis in different tumors $[34,35,54,68]$. In particular, our group reported that patients with high-grade osteosarcoma were associated to significant reduced survival when homozygous for 72Pro allele [35].

In the HGSOC here presented, if the identified variants, somatic and germline, were in cis configuration in the tumor, the driver TP53 splice mutation could have been coupled with the "pro-apoptotic" 72Arg allele, that may sustains the damaging effects of chemotherapy favoring tumor cell death. The particular trans configuration between the germline SNP and the somatic driver mutation suggests that it is critical for this tumor because it provides a selective advantage to cancer cells regardless of germline background. Somatic or germline variants have been mostly independently analyzed in cancer genome studies. Increasing evidences are focusing on the intricate linkage between germline and somatic variants which consequence is, from a molecular and phenotypic standpoint, the complex cancer disease, influenced by both inherited variants in germline DNA and somatic alterations acquired during formation of the tumor [69]. Germline variants are estimated, through genome-wide association studies [70], to contribute for a $20 \%$ to cancer development and have been reported to influence gene expression in tumors [69,71]. In ovarian cancer, a recent study integrated data from rare germline and somatic mutations identifying novel genes and variants of potential impact in ovarian cancer susceptibility [72]. Inactivation of tumor-suppressor genes and activation of oncogenes which finally lead to uncontrolled cell growth and metastatic spread, may depend on genetic alterations, including LOH and copy number variations (CNVs) [73]. In ovarian cancer, $\mathrm{LOH}$ of the WT allele is recognized as the tumor-initiating second hit in most of patients with a pathogenic germline mutation in $B R C A 1$ or $B R C A 2$ genes [72,74,75]. BRCA1 immunostaining has a negative predictive value of $100 \%$ for germline mutations in $B R C A 1$ (intact/positive staining corresponds to lack of $B R C A 1$ mutations) [76]. Furthermore, $94 \%$ of HGSOCs without homologous recombination (HR) alterations in DNA repair pathway (most commonly in BRCA1/2 genes) evaluated by NGS, exhibited positive BRCA1 expression [77]. We observed positive immunostaining for BRCA1 protein in the pre-NACT tumor sample from the HGSOC analyzed, suggesting the lack of germline (and probably somatic) BRCA1 alterations. This result is concordant with the unreported family history of ovarian and breast cancer and with unfavorable prognosis of the patient since carriers of either germline or somatic BRCA1 mutations are associated with improved survival compared to patients with non-BRCA mutations [78]. Furthermore, the somatic mutations in upstream HR modulators, such as in PTEN (included in the 26-cancer genes panel), that may induce a "BRCAness" phenotype [23], were not detected.

The cooperation between germline and somatic variants that often requires a close interaction to develop cancer is best exemplified by the "two-hit hypothesis" [79], in which a tumor suppressor gene is inactivated by the combination of an initial germline mutation of one allele, followed by the somatic inactivation of the other [80]. LOH, whereby the WT allele for a two-hit tumor suppressor is eliminated, has been implicated in many cancers, including ovarian [72]. Nevertheless, somatic LOH may result in the loss of either the variant or the reference allele in the tumor, respectively [26]. LOH analysis through the NGS approach can easily uncover heterozygous germline variants that are under potential selection in the tumor, one of the key indications is the significant increased or decreased VAF in the tumor sample [80]. Through the application of NGS technology, the comparison of SNP allele frequencies from paired tumor and normal DNA to evaluate whether heterozygous loci in the blood/normal reference DNA sample are turned into homozygous loci in the tumor DNA sample, represents a simple method to detect $\mathrm{LOH}[26,27,72,81]$. To investigate the possibility that somatic 
LOH could increase functional impact of the germline heterozygous SNV TP53 c.215C > G (p.Pro72Arg) detected in the tumor samples, we took advantage of the quantitative nature of NGS technique, which has a higher sensitivity than microsallite-based LOH and multiplex ligation-dependent probe amplification [82]. Although in the chemo-naïve HGSOC the VAF decrease for the informative germline SNV was significant and suggestive of $\mathrm{LOH}$, heterozygosity for the somatic mutation TP53 c.375+1G > A was clearly detected in the pre-NACT tumor sample by NGS and also by the less sensitive Sanger sequencing method. The pre-NACT tumor sample was probably not completely free of normal tissue and may have exhibited subclonal heterogeneity [27]. Notably, VAF $>70 \%$, related to only somatic mutations identified, was used to assess somatic LOH in 72 cases of primary HGSOC [55]: VAF related to the TP53 c.375+1G $>$ A somatic mutation overcome this threshold only in the post-NACT tumor sample. Overall, $\mathrm{LOH}$ was present in the post-NACT tumor sample with elimination, under the selective pressure of NACT, of the treatment-sensitive 72Arg allele and concomitant expansion of the variant/mutant allele of a driver splice site mutation in TP53 intron 4, in trans configuration with respect to the TP53 p.Pro72Arg SNP.

When arising in patients who are TP53 p.Pro72Arg germline heterozygotes, the 72Pro allele has been reported to be preferentially retained in primary ovarian [54] and breast [34] cancers, through LOH. Of note, in patients with chemo-naïve ovarian carcinoma, women homozygous for the 72Pro allele had a significant shorter survival compared to those who were carriers for an Arg allele: eighty-four percent of TP53 p.Pro72Arg germline heterozygotes showed somatic LOH (56\% retained 72Pro allele and 44\% retained 72Arg allele), but among the TP53 mutated tumors who exhibited LOH there was no preference in loss of either Pro or Arg allele [54]. Hence, this study reports for the first time, in one patient with HGSOC, a selective retention of the germline 72Pro allele after NACT treatment.

Clonal evolution has been studied in different malignancies [21,42,46,47,83-85]. Selective pressure induced by chemotherapy is exerted through the selection of mutations that confer proliferative or chemotherapy resistance advantages; thus, treatment-sensitive clones are eliminated, whereas refractory/resistant clones tend to become dominant $[86,87]$. In urothelial carcinoma, characterized by resistance to platinum-based treatment and rapid tumor progression, selective pressure exerted by chemotherapy has been investigated by analyzing (by NGS) matched sets of primary advanced tumors collected before and after chemo-treatment, demonstrating clonal enrichment in post-chemotherapy tumor samples $[42,83]$.

One important strength of this study is that, to our knowledge, it is the only investigation on clonal evolution of chemotherapy-refractory/resistant HGSOC after NACT, with relevance in translational medicine. The limitations of our study are due to the findings in only one case that should be explored and confirmed in other patients with HGSOC that undergo NACT based on platinum/paclitaxel therapy. Another limit is represented by the unexplored mutational profile in the metastatic sites, such as in pleural effusion or in abdominal ascites from this patient, and also from relapse/solid biopsies. Molecular profiling of different matched samples from the same patient may shed light on the comprehension of the mutagenic pressure from the primary untreated tumor, to the advanced and chemotherapy treated one, at different anatomic sites. Another possible application which could improve this type of study, is represented also by the characterization of the mutational profile in the liquid biopsies through analysis of circulating free DNA. Thus, further investigations are needed to provide new insights on the evolution of HGSOC under the neo-adjuvant interventions.

\section{Conclusions}

In summary, this study reports, in a patient with stage IV HGSOC characterized by poor response to treatment and prognosis, that the TP53 c.375+1G > A somatic mutation affecting a consensus SD site in intron 4 flanking region was an early mutational event, and it was shared in the tumor before and after NACT. SNV identified in the primary chemo-naïve HGSOC preceded clonal evolution exhibited in the tumor sample exposed to NACT, after the selective pressure of platinum/paclitaxel treatment. Expansion of the founder clone with the TP53 driver mutation through LOH was coupled with the 
retention of the 72Pro allele and the loss of the "pro-apoptotic" 72Arg allele, codified by the germline heterozygous SNP TP53 c.215C >G (p.Pro72Arg) in exon 4. LOH involving the 72Arg allele and the WT allele of the driver somatic SD mutation of TP53 in the resistant treated tumor, suggests that this event may play a role in chemoresistance. This dominant mutation in the resistant tumor should be considered as a clearly negative factor that may impact the patient's outcome. The assessment of the SNP TP53 c.215C >G should be also considered to verify the trans or cis pattern with respect to TP53 c.375+1G > A mutation and also over other splice mutations affecting directly and non-directly the SD site flanking exon 4 in TP53. Patients with trans configuration of these SNVs in the tumor are at high risk of mutation selection by NACT based on platinum/paclitaxel combination therapy. Our study highlights the interplay between germline and somatic variants under the selective pressure of NACT and may have a relevant impact in translational medicine.

Author Contributions: Conceptualization, M.G.; methodology, M.G., E.C., E.D.M., R.R., L.R., and V.C.; software, M.G., E.C., E.D.M., R.R. and F.E.; validation, M.G. and V.C.; formal analysis, M.G.; investigation, M.G., R.R., E.D.M., L.R., S.G., E.P., S.S., V.C., G.G., R.S., and E.C.; resources, G.T.; data curation, E.D.M., R.R., and F.E.; writing-original draft preparation, M.G.; writing—review and editing, M.G., G.G., V.C., and S.G.; visualization, M.G.; supervision, G.G. and V.C.; project administration, E.C. and G.T.; funding acquisition, G.T. and V.C.

Funding: This research was partially funded by the "2020-PHC2015 two-stage: comprehensive characterization and effective combinatorial targeting of HGSOC via single cell analysis" (Co-P.I. Dr. Vincenzo Canzonieri) and by the Italian Ministry of Health with $5 \times 1000$ funds.

Acknowledgments: We thank the Scientific and Administrative Directorates of Centro di Riferimento Oncologico (CRO)-Aviano for their kind support and Luigina Mei for her invaluable English language assistance.

Conflicts of Interest: The authors declare no conflict of interest. The funders had no role in the design of the study; in the collection, analyses, or interpretation of data; in the writing of the manuscript; or in the decision to publish the results.

\section{References}

1. Ferlay, J.; Colombet, M.; Soerjomataram, I.; Mathers, C.; Parkin, D.M.; Piñeros, M.; Znaor, A.; Bray, F. Estimating the global cancer incidence and mortality in 2018: GLOBOCAN sources and methods. Int. J. Cancer 2019, 144, 1941-1953. [CrossRef] [PubMed]

2. Wiedemeyer, W.R.; Beach, J.A.; Karlan, B.Y. Reversing Platinum Resistance in High-Grade Serous Ovarian Carcinoma: Targeting BRCA and the Homologous Recombination System. Front. Oncol. 2014, 4, 34. [CrossRef] [PubMed]

3. Siegel, R.L.; Miller, K.D.; Jemal, A. Cancer statistics, 2015. CA Cancer J. Clin. 2015, 65, 5-29. [CrossRef] [PubMed]

4. Associazione Italiana di Oncologia Medica (AIOM). Linee Guida Tumori dell'Ovaio 2018; AIOM: Italy, 2018; Available online: http://www.aiom.it/linee-guida-aiom-2018-tumori-dellovaio/ (accessed on 12 August 2019).

5. Winter, W.E.; Maxwell, G.L.; Tian, C.; Carlson, J.W.; Ozols, R.F.; Rose, P.G.; Markman, M.; Armstrong, D.K.; Muggia, F.; McGuire, W.P.; et al. Prognostic factors for stage III epithelial ovarian cancer: A Gynecologic Oncology Group Study. J. Clin. Oncol. 2007, 25, 3621-3627. [CrossRef] [PubMed]

6. Sato, S.; Itamochi, H. Neoadjuvant chemotherapy in advanced ovarian cancer: Latest results and place in therapy. Ther. Adv. Med. Oncol. 2014, 6, 293-304. [CrossRef]

7. Wright, A.A.; Bohlke, K.; Armstrong, D.K.; Bookman, M.A.; Cliby, W.A.; Coleman, R.L.; Dizon, D.S.; Kash, J.J.; Meyer, L.A.; Moore, K.N.; et al. Neoadjuvant Chemotherapy for Newly Diagnosed, Advanced Ovarian Cancer: Society of Gynecologic Oncology and American Society of Clinical Oncology Clinical Practice Guideline. J. Clin. Oncol. 2016, 34, 3460-3473. [CrossRef] [PubMed]

8. Luo, Y.; Lee, M.; Kim, H.S.; Chung, H.H.; Song, Y.S. Effect of neoadjuvant chemotherapy on platinum resistance in stage IIIC and IV epithelial ovarian cancer. Medicine 2016, 95, e4797. [CrossRef]

9. Vergote, I.; Tropé, C.G.; Amant, F.; Kristensen, G.B.; Ehlen, T.; Johnson, N.; Verheijen, R.H.; van der Burg, M.E.; Lacave, A.J.; Panici, P.B.; et al. Neoadjuvant chemotherapy or primary surgery in stage IIIC or IV ovarian cancer. N. Engl. J. Med. 2010, 363, 943-953. [CrossRef] 
10. Kehoe, S.; Hook, J.; Nankivell, M.; Jayson, G.C.; Kitchener, H.; Lopes, T.; Luesley, D.; Perren, T.; Bannoo, S.; Mascarenhas, M.; et al. Primary chemotherapy versus primary surgery for newly diagnosed advanced ovarian cancer (CHORUS): An open-label, randomised, controlled, non-inferiority trial. Lancet 2015, 386, 249-257. [CrossRef]

11. Melamed, A.; Fink, G.; Wright, A.A.; Keating, N.L.; Gockley, A.A.; Del Carmen, M.G.; Schorge, J.O.; Rauh-Hain, J.A. Effect of adoption of neoadjuvant chemotherapy for advanced ovarian cancer on all cause mortality: Quasi-experimental study. BMJ 2018, 360, j5463. [CrossRef]

12. Bristow, R.E.; Chi, D.S. Platinum-based neoadjuvant chemotherapy and interval surgical cytoreduction for advanced ovarian cancer: A meta-analysis. Gynecol. Oncol. 2006, 103, 1070-1076. [CrossRef] [PubMed]

13. Sehouli, J.; Savvatis, K.; Braicu, E.I.; Schmidt, S.C.; Lichtenegger, W.; Fotopoulou, C. Primary versus interval debulking surgery in advanced ovarian cancer: Results from a systematic single-center analysis. Int. J. Gynecol. Cancer 2010, 20, 1331-1340. [CrossRef] [PubMed]

14. Rosen, B.; Laframboise, S.; Ferguson, S.; Dodge, J.; Bernardini, M.; Murphy, J.; Segev, Y.; Sun, P.; Narod, S.A. The impacts of neoadjuvant chemotherapy and of debulking surgery on survival from advanced ovarian cancer. Gynecol. Oncol. 2014, 134, 462-467. [CrossRef] [PubMed]

15. Lim, M.C.; Song, Y.J.; Seo, S.S.; Yoo, C.W.; Kang, S.; Park, S.Y. Residual cancer stem cells after interval cytoreductive surgery following neoadjuvant chemotherapy could result in poor treatment outcomes for ovarian cancer. Oncol. Res. Treat. 2010, 33, 324-330. [CrossRef] [PubMed]

16. Matsuo, K.; Eno, M.L.; Im, D.D.; Rosenshein, N.B. Chemotherapy time interval and development of platinum and taxane resistance in ovarian, fallopian, and peritoneal carcinomas. Arch. Gynecol. Obstet. 2010, 281, 325-328. [CrossRef] [PubMed]

17. Cancer Genome Atlas Research Network. Integrated genomic analyses of ovarian carcinoma. Nature 2011, 474, 609-615. [CrossRef] [PubMed]

18. Kurman, R.J.; Shih, I.M. The Dualistic Model of Ovarian Carcinogenesis: Revisited, Revised, and Expanded. Am. J. Pathol. 2016, 186, 733-747. [CrossRef]

19. Ledermann, J.A.; Raja, F.A.; Fotopoulou, C.; Gonzales-Martin, A.; Colombo, N.; Sessa, C.; ESMO Guidelines Working Group. Newly diagnosed and relapsed epithelial ovarian carcinoma: ESMO Clinical Practice Guidelines for diagnosis, treatment and follow-up. Ann. Oncol. 2013, 24, vi24-vi32. [CrossRef]

20. Bashashati, A.; Ha, G.; Tone, A.; Ding, J.; Prentice, L.M.; Roth, A.; Rosner, J.; Shumansky, K.; Kalloger, S.; Senz, J.; et al. Distinct evolutionary trajectories of primary high-grade serous ovarian cancers revealed through spatial mutational profiling. J. Pathol. 2013, 231, 21-34. [CrossRef]

21. Patch, A.M.; Christie, E.L.; Etemadmoghadam, D.; Garsed, D.W.; George, J.; Fereday, S.; Nones, K.; Cowin, P.; Alsop, K.; Bailey, P.J.; et al. Whole-genome characterization of chemoresistant ovarian cancer. Nature 2015, 521, 489-494. [CrossRef]

22. Castellarin, M.; Milne, K.; Zeng, T.; Tse, K.; Mayo, M.; Zhao, Y.; Webb, J.R.; Watson, P.H.; Nelson, B.H.; Holt, R.A. Clonal evolution of high-grade serous ovarian carcinoma from primary to recurrent disease. J. Pathol. 2013, 229, 515-524. [CrossRef] [PubMed]

23. Garziera, M.; Roncato, R.; Montico, M.; De Mattia, E.; Gagno, S.; Poletto, E.; Scalone, S.; Canzonieri, V.; Giorda, G.; Sorio, R.; et al. New Challenges in Tumor Mutation Heterogeneity in Advanced Ovarian Cancer by a Targeted Next-Generation Sequencing (NGS) Approach. Cells 2019, 8, 584. [CrossRef] [PubMed]

24. Evans, T.; Matulonis, U. Next-Generation Sequencing: Role in Gynecologic Cancers. J. Natl. Compr. Cancer Netw. 2016, 14, 1165-1173. [CrossRef] [PubMed]

25. Garziera, M.; Cecchin, E.; Canzonieri, V.; Sorio, R.; Giorda, G.; Scalone, S.; De Mattia, E.; Roncato, R.; Gagno, S.; Poletto, E.; et al. Identification of Novel Somatic TP53 Mutations in Patients with High-Grade Serous Ovarian Cancer (HGSOC) Using Next-Generation Sequencing (NGS). Int. J. Mol. Sci. 2018, $19,1510$. [CrossRef]

26. Suarez-Kelly, L.P.; Akagi, K.; Reeser, J.W.; Samorodnitsky, E.; Reeder, M.; Smith, A.; Roychowdhury, S.; Symer, D.E.; Carson, W.E. Metaplastic breast cancer in a patient with neurofibromatosis type 1 and somatic loss of heterozygosity. Cold Spring Harb. Mol. Case Stud. 2018, 4, a002352. [CrossRef] [PubMed]

27. Lu, C.; Xie, M.; Wendl, M.C.; Wang, J.; McLellan, M.D.; Leiserson, M.D.; Huang, K.L.; Wyczalkowski, M.A.; Jayasinghe, R.; Banerjee, T.; et al. Patterns and functional implications of rare germline variants across 12 cancer types. Nat. Commun. 2015, 6, 10086. [CrossRef] [PubMed] 
28. Bouaoun, L.; Sonkin, D.; Ardin, M.; Hollstein, M.; Byrnes, G.; Zavadil, J.; Olivier, M. TP53 Variations in Human Cancers: New Lessons from the IARC TP53 Database and Genomics Data. Hum. Mutat. 2016, 37, 865-876. [CrossRef] [PubMed]

29. Flaman, J.M.; Frebourg, T.; Moreau, V.; Charbonnier, F.; Martin, C.; Chappuis, P.; Sappino, A.P.; Limacher, I.M.; Bron, L.; Benhattar, J.; et al. A simple p53 functional assay for screening cell lines, blood, and tumors. Proc. Natl. Acad. Sci. USA 1995, 92, 3963-3967. [CrossRef]

30. Smardova, J.; Liskova, K.; Ravcukova, B.; Malcikova, J.; Hausnerova, J.; Svitakova, M.; Hrabalkova, R.; Zlamalikova, L.; Stano-Kozubik, K.; Blahakova, I.; et al. Complex analysis of the p53 tumor suppressor in lung carcinoma. Oncol. Rep. 2016, 35, 1859-1867. [CrossRef]

31. Dumont, P.; Leu, J.J.; Della Pietra, A.C., III; George, D.L.; Murphy, M. The codon 72 polymorphic variants of p53 have markedly different apoptotic potential. Nat. Genet. 2003, 33, 357-365. [CrossRef]

32. Jeong, B.S.; Hu, W.; Belyi, V.; Rabadan, R.; Levine, A.J. Differential levels of transcription of p53-regulated genes by the arginine/proline polymorphism: p53 with arginine at codon 72 favors apoptosis. FASEB J. 2010, 24, 1347-1353. [CrossRef] [PubMed]

33. Zięba, S.; Kowalik, A.; Zalewski, K.; Rusetska, N.; Goryca, K.; Piaścik, A.; Misiek, M.; Bakuła-Zalewska, E.; Kopczyński, J.; Kowalski, K.; et al. Somatic mutation profiling of vulvar cancer: Exploring therapeutic targets. Gynecol. Oncol. 2018, 150, 552-561. [CrossRef]

34. Kyndi, M.; Alsner, J.; Hansen, L.L.; Sørensen, F.B.; Overgaard, J. LOH rather than genotypes of TP53 codon 72 is associated with disease-free survival in primary breast cancer. Acta Oncol. 2006, 45, 602-609. [CrossRef] [PubMed]

35. Toffoli, G.; Biason, P.; Russo, A.; De Mattia, E.; Cecchin, E.; Hattinger, C.M.; Pasello, M.; Alberghini, M.; Ferrari, C.; Scotlandi, K.; et al. Effect of TP53 Arg72Pro and MDM2 SNP309 polymorphisms on the risk of high-grade osteosarcoma development and survival. Clin. Cancer Res. 2009, 15, 3550-3556. [CrossRef] [PubMed]

36. Li, L.T.; Jiang, G.; Chen, Q.; Zheng, J.N. Ki67 is a promising molecular target in the diagnosis of cancer. Mol. Med. Rep. 2015, 11, 1566-1572. [CrossRef] [PubMed]

37. Lisio, M.A.; Fu, L.; Goyeneche, A.; Gao, Z.H.; Telleria, C. High-Grade Serous Ovarian Cancer: Basic Sciences, Clinical and Therapeutic Standpoints. Int. J. Mol. Sci. 2019, 20, 952. [CrossRef]

38. Taube, E.T.; Denkert, C.; Sehouli, J.; Kunze, C.A.; Dietel, M.; Braicu, I.; Letsch, A.; Darb-Esfahani, S. Wilms tumor protein 1 (WT1) - Not only a diagnostic but also a prognostic marker in high-grade serous ovarian carcinoma. Gynecol. Oncol. 2016, 140, 494-502. [CrossRef]

39. Köbel, M.; Bak, J.; Bertelsen, B.I.; Carpen, O.; Grove, A.; Hansen, E.S.; Levin Jakobsen, A.M.; Lidang, M.; Måsbäck, A.; Tolf, A.; et al. Ovarian carcinoma histotype determination is highly reproducible, and is improved through the use of immunohistochemistry. Histopathology 2014, 64, 1004-1013. [CrossRef]

40. Oaknin, A.; Guarch, R.; Barretina, P.; Hardisson, D.; González-Martín, A.; Matías-Guiu, X.; Pérez-Fidalgo, A.; Vieites, B.; Romero, I.; Palacios, J. Recommendations for biomarker testing in epithelial ovarian cancer: A National Consensus Statement by the Spanish Society of Pathology and the Spanish Society of Medical Oncology. Clin. Transl. Oncol. 2018, 20, 274-285. [CrossRef]

41. Netinatsunthorn, W.; Hanprasertpong, J.; Dechsukhum, C.; Leetanaporn, R.; Geater, A. WT1 gene expression as a prognostic marker in advanced serous epithelial ovarian carcinoma: An immunohistochemical study. BMC Cancer 2006, 6, 90. [CrossRef]

42. Faltas, B.M.; Prandi, D.; Tagawa, S.T.; Molina, A.M.; Nanus, D.M.; Sternberg, C.; Rosenberg, J.; Mosquera, J.M.; Robinson, B.; Elemento, O.; et al. Clonal evolution of chemotherapy-resistant urothelial carcinoma. Nat. Genet. 2016, 48, 1490-1499. [CrossRef] [PubMed]

43. Liu, D.; Abbosh, P.; Keliher, D.; Reardon, B.; Miao, D.; Mouw, K.; Weiner-Taylor, A.; Wankowicz, S.; Han, G.; Teo, M.Y.; et al. Mutational patterns in chemotherapy resistant muscle-invasive bladder cancer. Nat. Commun. 2017, 8, 2193. [CrossRef]

44. Arend, R.C.; Londoño, A.I.; Montgomery, A.M.; Smith, H.J.; Dobbin, Z.C.; Katre, A.A.; Martinez, A.; Yang, E.S.; Alvarez, R.D.; Huh, W.K.; et al. Molecular Response to Neoadjuvant Chemotherapy in High-Grade Serous Ovarian Carcinoma. Mol. Cancer Res. 2018, 16, 813-824. [CrossRef] [PubMed]

45. Sturm, I.; Bosanquet, A.G.; Hermann, S.; Güner, D.; Dörken, B.; Daniel, P.T. Mutation of p53 and consecutive selective drug resistance in B-CLL occurs as a consequence of prior DNA-damaging chemotherapy. Cell Death Differ. 2003, 10, 477-484. [CrossRef] [PubMed] 
46. Malcikova, J.; Stano-Kozubik, K.; Tichy, B.; Kantorova, B.; Pavlova, S.; Tom, N.; Radova, L.; Smardova, J.; Pardy, F.; Doubek, M.; et al. Detailed analysis of therapy-driven clonal evolution of TP53 mutations in chronic lymphocytic leukemia. Leukemia 2015, 29, 877-885. [CrossRef]

47. Amin, N.A.; Seymour, E.; Saiya-Cork, K.; Parkin, B.; Shedden, K.; Malek, S.N. A Quantitative Analysis of Subclonal and Clonal Gene Mutations before and after Therapy in Chronic Lymphocytic Leukemia. Clin. Cancer Res. 2016, 22, 4525-4535. [CrossRef] [PubMed]

48. Lønning, P.E.; Knappskog, S. Mapping genetic alterations causing chemoresistance in cancer: Identifying the roads by tracking the drivers. Oncogene 2013, 32, 5315-5330. [CrossRef] [PubMed]

49. Brachova, P.; Mueting, S.R.; Carlson, M.J.; Goodheart, M.J.; Button, A.M.; Mott, S.L.; Dai, D.; Thiel, K.W.; Devor, E.J.; Leslie, K.K. TP53 oncomorphic mutations predict resistance to platinum- and taxane-based standard chemotherapy in patients diagnosed with advanced serous ovarian carcinoma. Int. J. Oncol. 2015, 46, 607-618. [CrossRef]

50. Phillips, K.A.; Nichol, K.; Ozcelik, H.; Knight, J.; Done, S.J.; Goodwin, P.J.; Andrulis, I.L. Frequency of p53 mutations in breast carcinomas from Ashkenazi Jewish carriers of BRCA1 mutations. J. Natl. Cancer Inst. 1999, 91, 469-473. [CrossRef] [PubMed]

51. Ren, Z.P.; Ahmadian, A.; Pontén, F.; Nistér, M.; Berg, C.; Lundeberg, J.; Uhlén, M.; Pontén, J. Benign clonal keratinocyte patches with p53 mutations show no genetic link to synchronous squamous cell precancer or cancer in human skin. Am. J. Pathol. 1997, 150, 1791-1803. [PubMed]

52. Lu, M.L.; Wikman, F.; Orntoft, T.F.; Charytonowicz, E.; Rabbani, F.; Zhang, Z.; Dalbagni, G.; Pohar, K.S.; $\mathrm{Yu}, \mathrm{G}$; C Cordon-Cardo, C. Impact of alterations affecting the p53 pathway in bladder cancer on clinical outcome, assessed by conventional and array-based methods. Clin. Cancer Res. 2002, 8, 171-179. [PubMed]

53. Boonstra, J.J.; van der Velden, A.W.; Beerens, E.C.; van Marion, R.; Morita-Fujimura, Y.; Matsui, Y.; Nishihira, T.; Tselepis, C.; Hainaut, P.; Lowe, A.W.; et al. Mistaken identity of widely used esophageal adenocarcinoma cell line TE-7. Cancer Res. 2007, 67, 7996-8001. [CrossRef] [PubMed]

54. Galic, V.; Willner, J.; Wollan, M.; Garg, R.; Garcia, R.; Goff, B.A.; Gray, H.J.; Swisher, E.M. Common polymorphisms in TP53 and MDM2 and the relationship to TP53 mutations and clinical outcomes in women with ovarian and peritoneal carcinomas. Genes Chromosom. Cancer 2007, 46, 239-247. [CrossRef] [PubMed]

55. Cole, A.J.; Dwight, T.; Gill, A.J.; Dickson, K.A.; Zhu, Y.; Clarkson, A.; Gard, G.B.; Maidens, J.; Valmadre, S.; Clifton-Bligh, R.; et al. Assessing mutant p53 in primary high-grade serous ovarian cancer using immunohistochemistry and massively parallel sequencing. Sci. Rep. 2016, 6, 26191. [CrossRef]

56. Sveen, A.; Kilpinen, S.; Ruusulehto, A.; Lothe, R.A.; Skotheim, R.I. Aberrant RNA splicing in cancer; expression changes and driver mutations of splicing factor genes. Oncogene 2016, 35, 2413-2427. [CrossRef]

57. Hainaut, P.; Pfeifer, G.P. Somatic TP53 Mutations in the Era of Genome Sequencing. Cold Spring Harb. Perspect. Med. 2016, 6, a026179. [CrossRef]

58. Skinner, H.D.; Sandulache, V.C.; Ow, T.J.; Meyn, R.E.; Yordy, J.S.; Beadle, B.M.; Fitzgerald, A.L.; Giri, U.; Ang, K.K.; Myers, J.N. TP53 disruptive mutations lead to head and neck cancer treatment failure through inhibition of radiation-induced senescence. Clin. Cancer Res. 2012, 18, 290-300. [CrossRef]

59. Olivier, M.; Langerød, A.; Carrieri, P.; Bergh, J.; Klaar, S.; Eyfjord, J.; Theillet, C.; Rodriguez, C.; Lidereau, R.; Bièche, I.; et al. The clinical value of somatic TP53 gene mutations in 1794 patients with breast cancer. Clin. Cancer Res. 2006, 12, 1157-1167. [CrossRef]

60. Senturk, S.; Yao, Z.; Camiolo, M.; Stiles, B.; Rathod, T.; Walsh, A.M.; Nemajerova, A.; Lazzara, M.J.; Altorki, N.K.; Krainer, A.; et al. p53世 is a transcriptionally inactive p53 isoform able to reprogram cells toward a metastatic-like state. Proc. Natl. Acad. Sci. USA 2014, 111, E3287-E3296. [CrossRef]

61. Shirole, N.H.; Pal, D.; Kastenhuber, E.R.; Senturk, S.; Boroda, J.; Pisterzi, P.; Miller, M.; Munoz, G.; Anderluh, M.; Ladanyi, M.; et al. TP53 exon-6 truncating mutations produce separation of function isoforms with pro-tumorigenic functions. Elife 2017, 6, e25532. [CrossRef]

62. Smeby, J.; Sveen, A.; Eilertsen, I.A.; Danielsen, S.A.; Hoff, A.M.; Eide, P.W.; Johannessen, B.; Hektoen, M.; Skotheim, R.I.; Guren, M.G.; et al. Transcriptional and functional consequences of TP53 splice mutations in colorectal cancer. Oncogenesis 2019, 8, 35. [CrossRef] [PubMed]

63. Baptiste, N.; Friedlander, P.; Chen, X.; Prives, C. The proline-rich domain of p53 is required for cooperation with anti-neoplastic agents to promote apoptosis of tumor cells. Oncogene 2002, 21, 9-21. [CrossRef] [PubMed] 
64. Yu, H.; Chen, J.K.; Feng, S.; Dalgarno, D.C.; Brauer, A.W.; Schreiber, S.L. Structural basis for the binding of proline-rich peptides to SH3 domains. Cell 1994, 76, 933-945. [CrossRef]

65. Walker, K.K.; Levine, A.J. Identification of a novel p53 functional domain that is necessary for efficient growth suppression. Proc. Natl. Acad. Sci. USA 1996, 93, 15335-15340. [CrossRef]

66. Bonafé, M.; Ceccarelli, C.; Farabegoli, F.; Santini, D.; Taffurelli, M.; Barbi, C.; Marzi, E.; Trapassi, C.; Storci, G.; Olivieri, F.; et al. Retention of the p53 codon 72 arginine allele is associated with a reduction of disease-free and overall survival in arginine/proline heterozygous breast cancer patients. Clin. Cancer Res. 2003, 9, 4860-4864.

67. Xu, Y.; Yao, L.; Ouyang, T.; Li, J.; Wang, T.; Fan, Z.; Lin, B.; Lu, Y.; Xie, Y. p53 Codon 72 polymorphism predicts the pathologic response to neoadjuvant chemotherapy in patients with breast cancer. Clin. Cancer Res. 2005, 11, 7328-7333. [CrossRef]

68. Toyama, T.; Zhang, Z.; Nishio, M.; Hamaguchi, M.; Kondo, N.; Iwase, H.; Iwata, H.; Takahashi, S.; Yamashita, H.; Fujii, Y. Association of TP53 codon 72 polymorphism and the outcome of adjuvant therapy in breast cancer patients. Breast Cancer Res. 2007, 9, R34. [CrossRef]

69. Carter, H.; Marty, R.; Hofree, M.; Gross, A.M.; Jensen, J.; Fisch, K.M.; Wu, X.; DeBoever, C.; Van Nostrand, E.L.; Song, Y.; et al. Interaction Landscape of Inherited Polymorphisms with Somatic Events in Cancer. Cancer Discov. 2017, 7, 410-423. [CrossRef]

70. Galvan, A.; Ioannidis, J.P.; Dragani, T.A. Beyond genome-wide association studies: Genetic heterogeneity and individual predisposition to cancer. Trends Genet. 2010, 26, 132-141. [CrossRef]

71. Li, Q.; Seo, J.H.; Stranger, B.; McKenna, A.; Pe'er, I.; Laframboise, T.; Brown, M.; Tyekucheva, S.; Freedman, M.L. Integrative eQTL-based analyses reveal the biology of breast cancer risk loci. Cell 2013, 152, $633-641$. [CrossRef]

72. Kanchi, K.L.; Johnson, K.J.; Lu, C.; McLellan, M.D.; Leiserson, M.D.; Wendl, M.C.; Zhang, Q.; Koboldt, D.C.; Xie, M.; Kandoth, C.; et al. Integrated analysis of germline and somatic variants in ovarian cancer. Nat. Commun. 2014, 5, 3156. [CrossRef] [PubMed]

73. Chen, Y.; Chen, C. DNA copy number variation and loss of heterozygosity in relation to recurrence of and survival from head and neck squamous cell carcinoma: A review. Head Neck 2008, 30, 1361-1383. [CrossRef] [PubMed]

74. Weren, R.D.; Mensenkamp, A.R.; Simons, M.; Eijkelenboom, A.; Sie, A.S.; Ouchene, H.; van Asseldonk, M.; Gomez-Garcia, E.B.; Blok, M.J.; de Hullu, J.A.; et al. Novel BRCA1 and BRCA2 Tumor Test as Basis for Treatment Decisions and Referral for Genetic Counselling of Patients with Ovarian Carcinomas. Hum. Mutat. 2017, 38, 226-235. [CrossRef] [PubMed]

75. Khiabanian, H.; Hirshfield, K.M.; Goldfinger, M.; Bird, S.; Stein, M.; Aisner, J.; Toppmeyer, D.; Wong, S.; Chan, N.; Dhar, K.; et al. Inference of Germline Mutational Status and Evaluation of Loss of Heterozygosity in High-Depth, Tumor-Only Sequencing Data. JCO Precis. Oncol. 2018, 2018. [CrossRef] [PubMed]

76. Garg, K.; Levine, D.A.; Olvera, N.; Dao, F.; Bisogna, M.; Secord, A.A.; Berchuck, A.; Cerami, E.; Schultz, N.; Soslow, R.A. BRCA1 immunohistochemistry in a molecularly characterized cohort of ovarian high-grade serous carcinomas. Am. J. Surg. Pathol. 2013, 37, 138-146. [CrossRef] [PubMed]

77. Strickland, K.C.; Howitt, B.E.; Shukla, S.A.; Rodig, S.; Ritterhouse, L.L.; Liu, J.F.; Garber, J.E.; Chowdhury, D.; Wu, C.J.; D'Andrea, A.D.; et al. Association and prognostic significance of BRCA1/2-mutation status with neoantigen load, number of tumor-infiltrating lymphocytes and expression of PD-1/PD-L1 in high grade serous ovarian cancer. Oncotarget 2016, 7, 13587-13598. [CrossRef]

78. Bolton, K.L.; Chenevix-Trench, G.; Goh, C.; Sadetzki, S.; Ramus, S.J.; Karlan, B.Y.; Lambrechts, D.; Despierre, E.; Barrowdale, D.; McGuffog, L.; et al. Association between BRCA1 and BRCA2 mutations and survival in women with invasive epithelial ovarian cancer. JAMA 2012, 307, 382-390. [CrossRef]

79. Knudson, A.G., Jr. Mutation and cancer: Statistical study of retinoblastoma. Proc. Natl. Acad. Sci. USA 1971, 68, 820-823. [CrossRef]

80. Chen, J.; Weiss, W.A. Alternative splicing in cancer: Implications for biology and therapy. Oncogene 2015, 34, 1-14. [CrossRef]

81. de Jonge, M.M.; Ruano, D.; van Eijk, R.; van der Stoep, N.; Nielsen, M.; Wijnen, J.T.; Ter Haar, N.T.; Baalbergen, A.; Bos, M.E.M.M.; Kagie, M.J.; et al. Validation and Implementation of BRCA1/2 Variant Screening in Ovarian Tumor Tissue. J. Mol. Diagn. 2018, 20, 600-611. [CrossRef] 
82. Dubbink, H.J.; Atmodimedjo, P.N.; van Marion, R.; Krol, N.M.G.; Riegman, P.H.J.; Kros, J.M.; van den Bent, M.J.; Dinjens, W.N.M. Diagnostic Detection of Allelic Losses and Imbalances by Next-Generation Sequencing: 1p/19q Co-Deletion Analysis of Gliomas. J. Mol. Diagn. 2016, 18, 775-786. [CrossRef] [PubMed]

83. Vlachostergios, P.J.; Faltas, B.M. Treatment resistance in urothelial carcinoma: An evolutionary perspective. Nat. Rev. Clin. Oncol. 2018, 15, 495-509. [CrossRef] [PubMed]

84. Zenz, T.; Kröber, A.; Scherer, K.; Häbe, S.; Bühler, A.; Benner, A.; Denzel, T.; Winkler, D.; Edelmann, J.; Schwänen, C.; et al. Monoallelic TP53 inactivation is associated with poor prognosis in chronic lymphocytic leukemia: Results from a detailed genetic characterization with long-term follow-up. Blood 2008, 112, 3322-3329. [CrossRef] [PubMed]

85. Malcikova, J.; Smardova, J.; Rocnova, L.; Tichy, B.; Kuglik, P.; Vranova, V.; Cejkova, S.; Svitakova, M.; Skuhrova Francova, H.; Brychtova, Y.; et al. Monoallelic and biallelic inactivation of TP53 gene in chronic lymphocytic leukemia: Selection, impact on survival, and response to DNA damage. Blood 2009, 114, 5307-5314. [CrossRef] [PubMed]

86. Amirouchene-Angelozzi, N.; Swanton, C.; Bardelli, A. Tumor Evolution as a Therapeutic Target. Cancer Discov. 2017. [CrossRef] [PubMed]

87. Fedele, C.; Tothill, R.W.; McArthur, G.A. Navigating the challenge of tumor heterogeneity in cancer therapy. Cancer Discov. 2014, 4, 146-148. [CrossRef] [PubMed]

(C) 2019 by the authors. Licensee MDPI, Basel, Switzerland. This article is an open access article distributed under the terms and conditions of the Creative Commons Attribution (CC BY) license (http://creativecommons.org/licenses/by/4.0/). 